\title{
Structural Dynamics and Thermal Transport in Bismuth Chalcogenide Alloys
}

Jiayi Cen, ${ }^{1}$ Ioanna Pallikara ${ }^{1}$ and Jonathan M. Skelton ${ }^{l *}$

${ }^{1}$ Department of Chemistry, University of Manchester, Oxford Road, Manchester M13 9PL, UK

KEYWORDS thermoelectrics, bismuth chalcogenides, first-principles materials modelling, energetic and dynamical stability, lattice dynamics, thermal transport, alloys 


\section{ABSTRACT}

We present a detailed study of the structural dynamics, energetic and dynamical stability and thermal transport of the bismuth chalcogenides $\mathrm{Bi}_{2} \mathrm{~S}_{3}, \mathrm{Bi}_{2} \mathrm{Se}_{3}$ and $\mathrm{Bi}_{2} \mathrm{Te}_{3}$ and their alloys. The differing activities of the $\mathrm{Bi}$ cation lone pairs in $\mathrm{Bi}_{2} \mathrm{~S}_{3}, \mathrm{Bi}_{2} \mathrm{Se}_{3}$ and $\mathrm{Bi}_{2} \mathrm{Te}_{3}$ lead to competition between orthorhombic Pnma and rhombohedral $R \overline{3} m$ phases, with the latter favored by heavier chalcogen atoms, while the reported non-ambient phases of $\mathrm{Bi}_{2} \mathrm{Se}_{3}$ and $\mathrm{Bi}_{2} \mathrm{Te}_{3}$ show phonon instabilities under ambient conditions. The Pnma structure has weaker chemical bonding and stronger phonon anharmonicity than the $R \overline{3} m$ phase, resulting in an intrinsically lower lattice thermal conductivity. A thermodynamic model of $\mathrm{Bi}_{2}\left(\mathrm{Se}_{1-x} \mathrm{~S}_{x}\right)_{3}$ indicates that the $R \overline{3} m$ structure is energetically favored only for low S content, but the stability window can potentially be extended at lower formation temperatures. $\mathrm{Bi}_{2}\left(\mathrm{Se}_{1-x} \mathrm{Te}_{x}\right)_{3}$ in the $R \overline{3} m$ phase shows substantial deviation from ideal solid-solution behavior, due to a strong energetic preference for the Se and Te atoms to occupy the interior and exterior sites, respectively, in the constituent quintuple layers. Strain-field fluctuations induced by inhomogeneities in the chemical bonding are shown to play a significant role in determining the heat transport in the alloy systems, and chalcogen disorder away from the preferred symmetric structure is found to be an important factor in the reduced thermal conductivity of $\mathrm{Bi}_{2} \mathrm{SeTe}_{2}$ compared to the $\mathrm{Bi}_{2} \mathrm{Te}_{3}$ endpoint. The microscopic insight from these modelling studies provides new insight into the bismuth chalcogenides and their alloys, which may inform ongoing research to optimize the thermoelectric performance of these and related materials. 


\section{INTRODUCTION}

Rising concerns over climate change and dwindling non-renewable resources have led to increased emphasis on developing technologies to improve energy sustainability and to meet growing energy demand. Since more than $60 \%$ of the energy used worldwide is wasted as heat, ${ }^{1}$ thermoelectric generators (TEGs), which can extract electrical energy from a temperature gradient, are currently the subject of intensive research. ${ }^{2}$ TEGs are easily scalable to a range of sizes for applications including in the automotive, manufacturing and nuclear industries, and can thus potentially improve energy sustainability across a wide range of sectors. ${ }^{2}$

The performance of a thermoelectric material in a TEG is typically expressed by the dimensionless figure of merit $Z T:^{3}$

$$
Z T=\frac{S^{2} \sigma T}{\kappa_{\text {latt }}+\kappa_{\mathrm{el}}}
$$

The Seebeck coefficient $S$, electrical conductivity $\sigma$ and electronic thermal conductivity $\kappa_{\mathrm{el}}$ depend on the electronic structure of the material and can be fine-tuned by chemical doping. All three are interdependent through the carrier concentration $n$ and must be balanced to optimize the $Z T$ at a target operating temperature. The lattice thermal conductivity $\kappa_{\text {latt }}$ is the transport through phonons, which depends on the structure and bonding in the material and can in principle be optimized independently of the electrical properties. ${ }^{3-5}$ 
The requirement for a favorable electronic structure and low $\kappa_{\text {latt }}$ has led to a number of high-performance TEs based on "heavy" chalcogenides with strongly-anharmonic lattice dynamics and intrinsically low thermal conductivity. PbTe is generally considered as a standard for hightemperature thermoelectric applications and can reach a $Z T$ of 2.2 at $915 \mathrm{~K}$ with hierarchical nanostructuring. ${ }^{6}$ Its high performance has been attributed to anharmonic lattice dynamics ${ }^{7}$ and to a convergence of the electronic bands at elevated temperature leading to enhanced band degeneracy and a larger Seebeck coefficient. ${ }^{8,9}$ More recently, SnSe was shown to have a very high bulk $Z T$ of up to 2.6 at $923 \mathrm{~K}$, due to an ultra-low $\kappa_{\text {latt }}$ associated with the high-temperature phase transition. ${ }^{10-14}$ With a $Z T$ of $\sim 1.0$ between $300-400 \mathrm{~K}, \mathrm{Bi}_{2} \mathrm{Te}_{3}$ is the commercial standard for room-temperature TEGs at present, due to its favorable electronic properties and inherently low $\kappa_{\text {latt }}{ }^{2,15}$ the latter of which has been attributed to weak chemical bonding and highly anharmonic lattice dynamics. ${ }^{15,16}$

However, the low terrestrial abundance of Te (ca. $1 \mathrm{ppb}$ ) limits large-scale adoption. ${ }^{2}$ Therefore, there has been substantial investigation into variants of $\mathrm{Bi}_{2} \mathrm{Te}_{3}$ in the same tetradymite family ${ }^{17}$ (e.g. $\mathrm{Bi}_{2} \mathrm{Se}_{2} \mathrm{Te}$ ) and using strategies such as alloying to reduce or eliminate Te. ${ }^{18-22}$ Doping and alloying are common design strategies for enhancing the $Z T$ of thermoelectrics, as they can both improve the Seebeck coefficient by promoting band convergence and reduce the $\kappa_{\text {latt }}$ by introducing point defects that scatter phonons through mass- and strain-field fluctuations. ${ }^{3} \mathrm{~A}$ number of recent studies on bismuth chalcogenide alloy systems, including $\mathrm{Bi}_{2}(\mathrm{~S}, \mathrm{Se})_{3}, \mathrm{Bi}_{2}(\mathrm{~S}, \mathrm{Te})_{3}$ and $\mathrm{Bi}_{2}(\mathrm{Se}, \mathrm{Te})_{3}$, have reported a $Z T$ of $0.5-1.3$ (Table 1). ${ }^{19-24}$ 
Table 1 Reported thermoelectric figures of merit $Z T$ of some bismuth chalcogenide alloys.

\begin{tabular}{lcc}
\hline & $Z T$ & $T[\mathrm{~K}]^{\mathrm{a}}$ \\
\hline $\mathrm{Bi}_{2}\left(\mathrm{STe}_{2}\right)^{19}$ & 0.8 & 573 \\
\hline $\mathrm{Bi}_{2}\left(\mathrm{~S}_{2} \mathrm{Se}^{19}\right.$ & 0.8 & 573 \\
\hline $\mathrm{Bi}_{2}\left(\mathrm{Se}_{0.3} \mathrm{Te}_{2.7}\right)^{20,23}$ & $0.54 / 1.27$ & $\mathrm{RT}$ \\
\hline $\mathrm{Bi}_{2}\left(\mathrm{Se}_{0.5} \mathrm{Te}_{2.5}\right)^{21}$ & 1.28 & $\mathrm{RT}$ \\
\hline $\mathrm{Bi}_{2}\left(\mathrm{Se}_{0.6} \mathrm{Te}_{2.4}\right)^{24}$ & 0.79 & $\mathrm{RT}$ \\
\hline $\mathrm{Bi}_{2}\left(\mathrm{Se}_{1.5} \mathrm{Te}_{1.5}\right)^{22}$ & 0.67 & 473 \\
\hline
\end{tabular}

${ }^{\mathrm{a}} \mathrm{RT}$ - room temperature.

Liu et al. recently carried out a systematic study of the $\mathrm{Bi}_{2}(\mathrm{~S}, \mathrm{Se}, \mathrm{Te})_{3}$ ternary phase space and established several compositional trends in the thermoelectric performance. ${ }^{19}$ The thermal conductivities of mixed compositions along the $\mathrm{Bi}_{2}\left(\mathrm{Se}_{1-x} \mathrm{Te}_{x}\right)_{3}$ tie line were found to be lower than the closest endpoints and to reach a minimum for $\mathrm{Bi}_{2} \mathrm{Se}_{2} \mathrm{Te}$. $\mathrm{Bi}_{2} \mathrm{STe}_{2}$ and $\mathrm{Bi}_{2} \mathrm{~S}_{2} \mathrm{Te}$ were found to show lower thermal conductivities than either of $\mathrm{Bi}_{2} \mathrm{~S}_{3}$ and $\mathrm{Bi}_{2} \mathrm{Te}_{3}$, and a global minimum in the $\kappa_{\text {latt }}$ was identified at $\mathrm{Bi}_{2} \mathrm{Te}_{1.2} \mathrm{Se}_{1.8}$. The reduced $\kappa_{\text {latt }}$ of the mixed compositions was attributed to enhanced phonon scattering in the alloys. In contrast, measurements of the electrical properties showed a strong dependence on the Te content, and the weighted mobility was optimized towards $\mathrm{Bi}_{2} \mathrm{Te}_{3}$.

Thermoelectrics are attractive candidates for modelling studies because the terms in Eq. 1 are amenable to calculation. The electrical properties can be predicted using semi-classical Boltzmann transport theory, ${ }^{25,26}$ and it has also recently become possible to use first-principles modelling to investigate the structural dynamics and lattice thermal conductivity. ${ }^{27-30}$ The latter calculations have played an important role in understanding the intrinsic anharmonicity in materials with low $\kappa_{\text {latt }},{ }^{11,14,31-34}$ including several flagship thermoelectrics, ${ }^{11,12,14,33}$ and have also 
been used to identify and characterize the thermal transport in novel candidate TEs. ${ }^{34}$ There are several reports in the literature of modelling studies on the thermoelectric properties of bulk bismuth chalcogenides ${ }^{35-39}$ and nanostructures (e.g. single layers), ${ }^{40-43}$ including a handful of studies that investigate the structural dynamics and thermal conductivity. ${ }^{36,40-43}$ Modelling studies on the alloys are more scarce, owing to the higher complexity of the calculations, and tend to be restricted to compositions with well-defined structures..$^{37,41,42}$

Previous theoretical studies of alloy systems using first-principles density functional theory (DFT) and statistical thermodynamics have demonstrated the capacity systematically to explore a range of compositions between the endpoints of a two-component alloy system. ${ }^{44,45}$ The structure, properties and energetic and dynamical stability of different compositions can be evaluated in silico, providing a means to predict the electronic structure and thermal transport of the alloy and to guide experimental design towards optimizing the properties for thermoelectric applications. ${ }^{44}$ 46 The high computational resource requirements for modelling the lattice dynamics and thermal transport, however, pose significant challenges to studying alloy systems this way, which has resulted in few investigations in this direction. ${ }^{42,44-48}$

In this work, we use first-principles DFT calculations to perform a comprehensive study of the lattice dynamics, energetic and dynamical stability and thermal transport of all of the reported phases of $\mathrm{Bi}_{2} \mathrm{~S}_{3}, \mathrm{Bi}_{2} \mathrm{Se}_{3}$ and $\mathrm{Bi}_{2} \mathrm{Te}_{3}$, and we employ methodology developed in previous studies to investigate the thermodynamics and properties of $\mathrm{Bi}_{2}(\mathrm{Se}, \mathrm{S})_{3}$ and $\mathrm{Bi}_{2}(\mathrm{Se}, \mathrm{Te})_{3}$ alloys. The differing activities of the Bi cation lone pairs in $\mathrm{Bi}_{2} \mathrm{~S}_{3}, \mathrm{Bi}_{2} \mathrm{Se}_{3}$ and $\mathrm{Bi}_{2} \mathrm{Te}_{3}$ lead to competition between $R \overline{3} m$ and Pnma phases with very different thermal transport properties, which we attribute quantitatively to differences in the phonon group velocities and lifetimes. Whereas the $\mathrm{Bi}_{2}(\mathrm{Se}, \mathrm{S})_{3}$ alloy in the Pnma phase forms a homogenous solid solution, the $\mathrm{Bi}_{2}(\mathrm{Se}, \mathrm{S})_{3}$ and $\mathrm{Bi}_{2}(\mathrm{Se}, \mathrm{Te})_{3}$ alloys in the $R \overline{3} m$ 
phase show strong structural preferences for certain chalcogen arrangements. This leads to significant deviation from ideal mixing behavior, which can also be rationalized in terms of electrostatics. Finally, we use a variety of models to investigate the thermal conductivity in the alloys, and we show that the strain field introduced by inhomogeneous chemical bonding is likely to have a significant impact on the transport. The detailed microscopic insight from these studies highlights the benefits of this theoretical modelling approach for studying alloy systems, providing the potential for its extension to a wider range of solid-state materials for thermoelectric applications.

\section{METHODS}

First-principles calculations were carried out using pseudopotential plane-wave DFT as implemented in the Vienna ab initio Simulation Package (VASP) code. ${ }^{49,50}$ Initial structures of the Pnma, $R \overline{3} m$, and $P 4_{2} / n m c$ phases of the bismuth chalcogenides were obtained from the Materials Project $^{51}$ database and fully optimized using a plane-wave cutoff of $600 \mathrm{eV}$ and the $\Gamma$-centred Monkhorst-Pack k-point sampling meshes ${ }^{52}$ listed in Table 2. These parameters were determined to converge the total energy to $<1 \mathrm{meV}^{-1}$ atom $^{-1}$ and the pressure to $<1 \mathrm{kbar}(0.1 \mathrm{GPa})$. The Materials Project has two entries for $R \overline{3} m \mathrm{Bi}_{2} \mathrm{Te}_{3}$, the second of which has a different ordering of the atoms within the quintuple layers, and which we denote $R \overline{3} m^{*}$. The PBEsol GGA functional ${ }^{53}$ with the DFT-D3 dispersion correction ${ }^{54}$ (i.e. PBEsol+D3) was used to model exchange and correlation effects. PAW pseudopotentials ${ }^{50,55}$ were used to model the core electrons, with the valence electrons of $\mathrm{S}, \mathrm{Se}$, and $\mathrm{Te}$ and the valence and semi-core $d$ electrons of $\mathrm{Bi}$ treated as valence states. Tolerances of $10^{-8} \mathrm{eV}$ and $10^{-2} \mathrm{eV} \AA^{-1}$ were applied to total energy and forces during wavefunction and geometry optimisation, respectively. 
Table 2 k-point sampling, supercells used to compute the $2^{\text {nd }}$-order and $3^{\text {rd }}$-order interatomic force constants (IFCs), and q-point meshes used to evaluate the thermal conductivity for the eight bismuth chalcogenide phases examined in this work.

\begin{tabular}{lcccc}
\hline & & \multicolumn{2}{c}{ Phonon Supercells (\# Atoms) } & \\
\cline { 3 - 4 } System & k-Point Sampling ${ }^{\text {a }}$ & $2^{\text {nd }}$-Order IFCs & $3^{\text {rd }}$-Order IFCs & q-point Sampling ${ }^{\mathrm{b}}$ \\
\hline $\mathrm{Bi}_{2} \mathrm{~S}_{3}(R-3 m)$ & $4 \times 4 \times 4$ & $4 \times 4 \times 4(320)$ & $2 \times 2 \times 2(40)$ & $14 \times 14 \times 14$ \\
\hline $\mathrm{Bi}_{2} \mathrm{~S}_{3}(P n m a)$ & $6 \times 2 \times 2$ & $3 \times 1 \times 1(60)$ & $2 \times 1 \times 1(40)$ & $12 \times 4 \times 4$ \\
\hline $\mathrm{Bi}_{2} \mathrm{Se}_{3}(R-3 m)$ & $6 \times 6 \times 6$ & $4 \times 4 \times 4(320)$ & $2 \times 2 \times 2(40)$ & $14 \times 14 \times 14$ \\
\hline $\mathrm{Bi}_{2} \mathrm{Se}_{3}(P n m a)$ & $1 \times 6 \times 2$ & $1 \times 3 \times 1(60)$ & $1 \times 2 \times 1(40)$ & $4 \times 12 \times 4$ \\
\hline $\mathrm{Bi}_{2} \mathrm{Se}_{3}\left(P 4{ }_{2} / n m c\right)$ & $2 \times 2 \times 1$ & $2 \times 2 \times 2(320)$ & - & - \\
\hline $\mathrm{Bi}_{2} \mathrm{Te}_{3}(R-3 m)$ & $4 \times 4 \times 4$ & $4 \times 4 \times 4(320)$ & $2 \times 2 \times 2(40)$ & $14 \times 14 \times 14$ \\
\hline $\mathrm{Bi}_{2} \mathrm{Te}_{3}\left(R-3 m^{*}\right)$ & $11 \times 11 \times 11$ & $2 \times 2 \times 2(40)$ & - & - \\
\hline $\mathrm{Bi}_{2} \mathrm{Te}_{3}(P n m a)$ & $2 \times 9 \times 3$ & $1 \times 3 \times 1(60)$ & $1 \times 2 \times 1(40)$ & $4 \times 12 \times 4$ \\
\hline
\end{tabular}

${ }^{a} \mathbf{k}$-point meshes are given as the number of subdivisions along the three reciprocal lattice vectors in a $\Gamma$-centered Monkhorst-Pack mesh. ${ }^{\mathrm{b}} \mathbf{q}$-point meshes are given as regular $\Gamma$-centered meshes.

The size of the charge-density grids was set automatically to avoid aliasing errors, the PAW projections were performed in reciprocal space, and non-spherical contributions to the gradient corrections inside the PAW spheres were accounted for.

Lattice dynamics calculations were performed on the optimized structures to obtain the $2^{\text {nd }}$ and $3^{\text {rd }}$-order interatomic force constants (IFCs) using the supercell finite-displacement approach implemented in the Phonopy and Phono3py packages. ${ }^{27,28}$ The IFCs were determined using the supercells shown in Table 2 and displacement distances of $10^{-2}$ and $3 \times 10^{-2} \AA$ for the harmonic and anharmonic calculations respectively. Force calculations were performed using VASP. The kpoint meshes in Table 2 were reduced proportionally for the chosen supercells, and additional charge-density grids with $8 \times$ the number points as the standard grids were used to ensure accurate forces. Phonon density of states (DoS) curves were obtained using Fourier interpolation to evaluate 
frequencies on uniform $48 \times 48 \times 48 \Gamma$-centered q-point meshes and the liner tetrahedron method for Brillouin Zone integration. The phonon contributions to the constant-volume (Helmholtz) free energy were also evaluated using these sampling meshes. Phonon dispersions were computed by interpolating phonon frequencies along strings of high symmetry q-points, with the band paths determined using the SeeK-path code. ${ }^{56}$ Thermal conductivity calculations were performed within the single-mode relaxation-time approximation (RTA). ${ }^{28}$ The modal properties were sampled on the regular $\Gamma$-centered $\mathbf{q}$-point meshes in Table 2. These were sufficient to converge the calculated lattice thermal conductivities to within $5 \%$, except for $P n m a \mathrm{Bi}_{2} \mathrm{Se}_{3}$, which due to erratic convergence was converged to within $\sim 10 \%$.

Solid solution models of Pnma $\mathrm{Bi}_{2}\left(\mathrm{~S}_{1-x} \mathrm{Se}_{x}\right)_{3}$ and $R \overline{3} m \mathrm{Bi}_{2}\left(\mathrm{~S}_{1-x} \mathrm{Se}_{x}\right)_{3}$ and $\mathrm{Bi}_{2}\left(\mathrm{Se}_{1-x} \mathrm{Te}_{x}\right)_{3}$ were constructed in single cells and $2 \times 2 \times 1$ supercells of one of the two endpoints. The Transformer $\operatorname{code}^{57}$ was used to evaluate the symmetry-inequivalent structures and associated degeneracies obtained by successive substitutions of the chalcogen atoms between the two endpoints. This yielded 1,072 and 378 unique structures in 13 compositions for the Pnma and $R \overline{3} m$ alloys, respectively. The 560 structures in every other composition in the Pnma $\mathrm{Bi}_{2}\left(\mathrm{~S}_{1-x} \mathrm{Se}_{x}\right)_{3}$ alloy and the combined 756 structures in the two $R \overline{3} m$ alloys were optimized, and the total energies combined with the degeneracies to construct thermodynamic partition functions, investigate the mixing thermodynamics, and to compute statistical averages of properties. The $\mathbf{k}$-point sampling was set to $2 \times 6 \times 2$ for Pnma $\mathrm{Bi}_{2}\left(\mathrm{~S}_{1-x} \mathrm{Se}_{x}\right)_{3}$ and $3 \times 3 \times 6$ for $R \overline{3} m \mathrm{Bi}_{2}\left(\mathrm{~S}_{1-x} \mathrm{Se}_{x}\right)_{3}$ and $\mathrm{Bi}_{2}\left(\mathrm{Se}_{1-x} \mathrm{Te}_{x}\right)_{3}$.

Finally, we also performed phonon and thermal-conductivity calculations on two selected structures in the $\mathrm{Bi}_{2}\left(\mathrm{Se}_{1-x} \mathrm{Te}_{x}\right)$ alloy, with the $2^{\text {nd }}$-order and $3^{\text {rd }}$-order IFCs determined in supercells matching those used for the endpoint structures. 


\section{RESULTS AND DISCUSSION}

Chemical and Lattice Stability of the Bismuth Chalcogenides. we identified four reported crystal phases across the three chalcogenides $\mathrm{Bi}_{2} \mathrm{~S}_{3}, \mathrm{Bi}_{2} \mathrm{Se}_{3}$ and $\mathrm{Bi}_{2} \mathrm{Te}_{3}$ (Figure 1). $\mathrm{Bi}_{2} \mathrm{~S}_{3}$ is reported in an orthorhombic Pnma phase with 20 atoms in the primitive cell. ${ }^{19} \mathrm{Bi}_{2} \mathrm{Se}_{3}$ is reported in an equivalent Pnma phase and rhombohedral $R \overline{3} m$ and tetragonal $P 4_{2} / n m c$ phases with five and 40 atoms in the respective primitive cells. ${ }^{19,58-60}$ Finally, two $R \overline{3} m$ phases of $\mathrm{Bi}_{2} \mathrm{Te}_{3}$ are reported, both with five atoms in the primitive cell; ${ }^{19,61}$ the first of these is isostructural with the $R \overline{3} m$ phase of $\mathrm{Bi}_{2} \mathrm{Se}_{3}$, while the second, which we denote $R \overline{3} m^{*}$, has different ordering of atoms within the quintuple layers along the crystallographic $c$ axis.

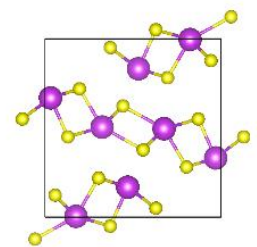

Pnma $\mathrm{Bi}_{2} \mathrm{~S}_{3}$

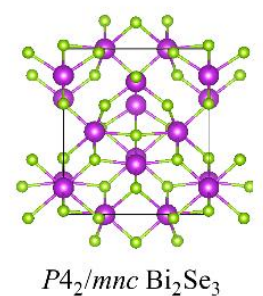

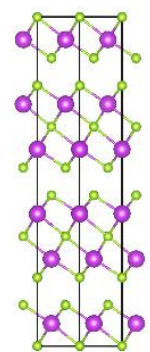

$R \overline{3} m \mathrm{Bi}_{2} \mathrm{Se}_{3}$

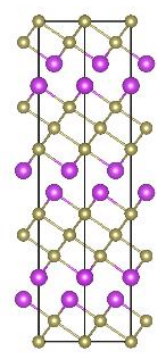

$R \overline{3} m * \mathrm{Bi}_{2} \mathrm{Te}_{3}$

Figure 1 Representative structures of the four bismuth chalcogenide phases examined in this work:

Pnma $\mathrm{Bi}_{2} \mathrm{~S}_{3}, P 4_{2} / n m c$ and $R \overline{3} m \mathrm{Bi}_{2} \mathrm{Se}_{3}$, and $R \overline{3} m * \mathrm{Bi}_{2} \mathrm{Te}_{3}$. The Bi atoms are colored purple and the $\mathrm{S}$, Se and Te atoms are colored yellow, green and olive, respectively. These images were prepared using the VESTA software. ${ }^{62}$ 
Since the Pnma and $R \overline{3} m$ phases are common to more than one chalcogenide, we also performed calculations on $\mathrm{Bi}_{2} \mathrm{Te}_{3}$ in the Pnma structure and $\mathrm{Bi}_{2} \mathrm{~S}_{3}$ in the $R \overline{3} m$ structure. In total, we therefore investigated eight structures: two of $\mathrm{Bi}_{2} \mathrm{~S}_{3}$, and three each of $\mathrm{Bi}_{2} \mathrm{Se}_{3}$ and $\mathrm{Bi}_{2} \mathrm{Te}_{3}$.

The optimized lattice parameters and relative lattice energies $\Delta U_{\text {latt }}$ are shown in Table 3. The lattice parameters are in good agreement with available measurements, ${ }^{19,59-61}$ suggesting that PBEsol+D3 provides a good description of the structures. With the exception of $P 4_{2} / n m c \mathrm{Bi}_{2} \mathrm{Se}_{3}$ and $R \overline{3} m * \mathrm{Bi}_{2} \mathrm{Te}_{3}$, the calculated and experimental values are within $4 \%$. For these two structures, a larger deviation of $\sim 10 \%$ is observed, which can be explained by the fact that these phases are prepared under high-pressure and high-temperature (i.e. non-ambient) synthesis conditions..$^{60,61}$ For $\mathrm{Bi}_{2} \mathrm{~S}_{3}$, the calculations predict the Pnma phase to be more stable than the $R \overline{3} m$ phase by $2.9 \mathrm{~kJ}$ $\mathrm{mol}^{-1}$ atom ${ }^{-1}$. For $\mathrm{Bi}_{2} \mathrm{Se}_{3}$, the stability is reversed, with the $R \overline{3} m$ being more stable than the Pnma phase by $1.8 \mathrm{~kJ} \mathrm{~mol}^{-1}$ atom ${ }^{-1}$. The $P 4_{2} / n m c$ phase is predicted to be much higher in energy than either of these, which we again ascribe to it being prepared under non-ambient conditions. The calculations again predict the $R \overline{3} m$ phase of $\mathrm{Bi}_{2} \mathrm{Te}_{3}$ to be more stable than the Pnma phase, this time by a larger energy difference of $5.6 \mathrm{~kJ} \mathrm{~mol}^{-1}$ atom $^{-1}$. This is small enough that the Pnma phase may be accessible, but there are no experimental reports of its synthesis. As for the non-ambient $P 4_{2} / n m c$ phase of $\mathrm{Bi}_{2} \mathrm{Se}_{3}$, the $R \overline{3} m *$ phase of $\mathrm{Bi}_{2} \mathrm{Te}_{3}$ is predicted to be considerably higher in energy than both the $R \overline{3} m$ and Pnma phases. This can be understood by the different atomic ordering in the quintuple layers: in the $R \overline{3} m$ phase, layers of $\mathrm{Bi}^{3+}$ are sandwiched by oppositelycharged layers of $\mathrm{Te}^{2-}$ layers, which is electrostatically favorable, whereas the $R \overline{3} m^{*}$ phase has adjacent layers of $\mathrm{Te}$ atoms that depending on the electron density distribution may result in less favorable electrostatic interactions and/or some level of electrostatic repulsion. 
The energetic preference of the heavier chalcogenides to adopt the $R \overline{3} m$ structure can be rationalized by the stereochemical activity of the $\mathrm{Bi} 6 \mathrm{~s}$ lone pair. $\mathrm{Bi}^{3+}$ experiences an inert-pair effect due to the relativistic contraction of the $6 \mathrm{~s}$ orbital. Interactions between the $\mathrm{Bi} 6 \mathrm{~s}$ and anion $\mathrm{p}$ orbitals produces a high-energy antibonding orbitals that can interact with the $\mathrm{Bi} 6 \mathrm{p}$, thus indirectly mediating Bi $6 \mathrm{~s} / 6 \mathrm{p}$ hybridization. ${ }^{63}$ Since this $\mathrm{s} / \mathrm{p}$ mixing is forbidden by symmetry in an octahedral bonding environment, a structural distortion is required that results in the lone pair occupying a coordination site. The distortion is only favored when the stabilization due to the anion-mediated $\mathrm{s} / \mathrm{p}$ mixing offsets the loss of electrostatic stabilization due to the reduced coordination number. The orbital interaction between the Bi $6 \mathrm{~s}$ and $\mathrm{S} \mathrm{3p}$ orbitals is the strongest among the three bismuth chalcogenides, as the relative energy difference is the smallest. $\mathrm{Bi}_{2} \mathrm{~S}_{3}$ therefore preferentially adopts the low-symmetry orthorhombic Pnma phase with an active lone pair, and remains stable even under pressure. ${ }^{64,65}$

Table 3 Optimized lattice parameters, relative lattice energies $\Delta U_{\text {latt }}$ and dynamical stabilities of the eight bismuth chalcogenide phases considered in this work.

\begin{tabular}{|c|c|c|c|c|c|}
\hline & $a^{\mathrm{a}}[\AA]$ & $b^{\mathrm{a}}[\AA]$ & $c^{\mathrm{a}}[\AA]$ & $\begin{array}{c}\Delta U_{\text {latt }^{\mathrm{b}}} \\
{\left[\mathrm{kJmol}^{-1} \text { atom }^{-1}\right]}\end{array}$ & $\begin{array}{c}\text { Dynamically } \\
\text { stable? }\end{array}$ \\
\hline $\mathrm{Bi}_{2} \mathrm{~S}_{3}(P n m a)$ & $3.94(3.98)$ & $10.89(11.14)$ & $11.05(11.29)$ & 0.0 & Yes \\
\hline $\mathrm{Bi}_{2} \mathrm{~S}_{3}(R \overline{3} m)$ & 3.98 & - & 26.77 & 2.9 & Yes \\
\hline $\mathrm{Bi}_{2} \mathrm{Se}_{3}($ Pnma $)$ & $11.76(11.71)$ & $4.08(4.14)$ & $11.26(11.61)$ & 1.8 & Yes \\
\hline $\mathrm{Bi}_{2} \mathrm{Se}_{3}(R \overline{3} m)$ & $4.12(4.14)$ & - & $27.72(28.63)$ & 0.0 & Yes \\
\hline $\begin{array}{l}\mathrm{Bi}_{2} \mathrm{Se}_{3} \\
\left(P 4_{2} / n m c\right)\end{array}$ & $10.05(9.23)$ & - & $11.48(12.70)$ & 16.8 & No \\
\hline $\mathrm{Bi}_{2} \mathrm{Te}_{3}($ Pnma $)$ & 12.30 & 4.32 & 12.13 & 5.6 & Yes \\
\hline $\mathrm{Bi}_{2} \mathrm{Te}_{3}(R \overline{3} m)$ & $4.37(4.39)$ & - & $29.55(30.50)$ & 0.0 & Yes \\
\hline $\mathrm{Bi}_{2} \mathrm{Te}_{3}\left(R \overline{3} m^{*}\right)$ & $4.49(4.42)$ & - & $27.05(29.84)$ & 19.6 & No \\
\hline
\end{tabular}

${ }^{a}$ Experimental data from Refs. ${ }^{19,59-61}$ shown in parentheses for comparison. ${ }^{\mathrm{b}}$ Lattice energies reported relative to the most stable phase of each chalcogenide. 
On the other hand, the poorer energy match between the Bi $6 s$ and Se $4 p / T e ~ 5 p$ leads to weaker orbital interactions that are insufficient to stabilize an active lone pair, resulting in "quenching". ${ }^{66}$ $\mathrm{Bi}_{2} \mathrm{Se}_{3}$ and $\mathrm{Bi}_{2} \mathrm{Te}_{3}$ therefore preferentially adopt the higher-symmetry $R \overline{3} m$ phase.

Figure 2 shows representative phonon dispersion and density-of-states (DoS) curves of each of the four structures, viz. Pnma $\mathrm{Bi}_{3} \mathrm{~S}_{3}, R \overline{3} m$ and $P 4_{2} / n m c \mathrm{Bi}_{2} \mathrm{Se}_{3}$, and $R \overline{3} m * \mathrm{Bi}_{2} \mathrm{Te}_{3}$. Data for all eight of the structures listed in Table 3 are given in the Figures S1-S8. The 20 and 5 atoms in the Pnma and $R \overline{3} m$ primitive cells lead to 60 and 15 bands in at each phonon wavevector. The phonon DoS curves show a clear separation of the modes into low- and high-frequency groups separated by a narrow "phonon bandgap". The low-frequency modes are predominantly associated with the Bi atoms, while the higher-frequency modes are associated with the chalcogen atoms. The two therefore appear in a 2:3 ratio. None of the Pnma or $R \overline{3} m$ dispersions show imaginary modes, indicating that these structures are dynamically stable phases for all three chalcogenides. On the other hand, the dispersions of $P 4_{2} / n m c \mathrm{Bi}_{2} \mathrm{Se}_{3}$ and $R \overline{3} m^{*} \mathrm{Bi}_{2} \mathrm{Te}_{3}$ both show prominent imaginary modes, indicating that these structures are dynamically unstable. Taken together with their relatively high energies, we therefore conclude that these phases are highly unlikely to be formed under ambient conditions. The dynamical stabilities of the eight structures, assessed from the presence or absence of imaginary modes in the calculated phonon dispersions, are collected alongside the relative energies in Table 3.

We can further use the phonon calculations to compute the vibrational contributions to the temperature-dependent constant-volume (Helmholtz) free energy $A$ :

$$
A(T)=U^{\mathrm{latt}}+A^{\mathrm{vib}}(T)=U^{\mathrm{latt}}+U^{\mathrm{vib}}(T)-T S^{\mathrm{vib}}(T)
$$



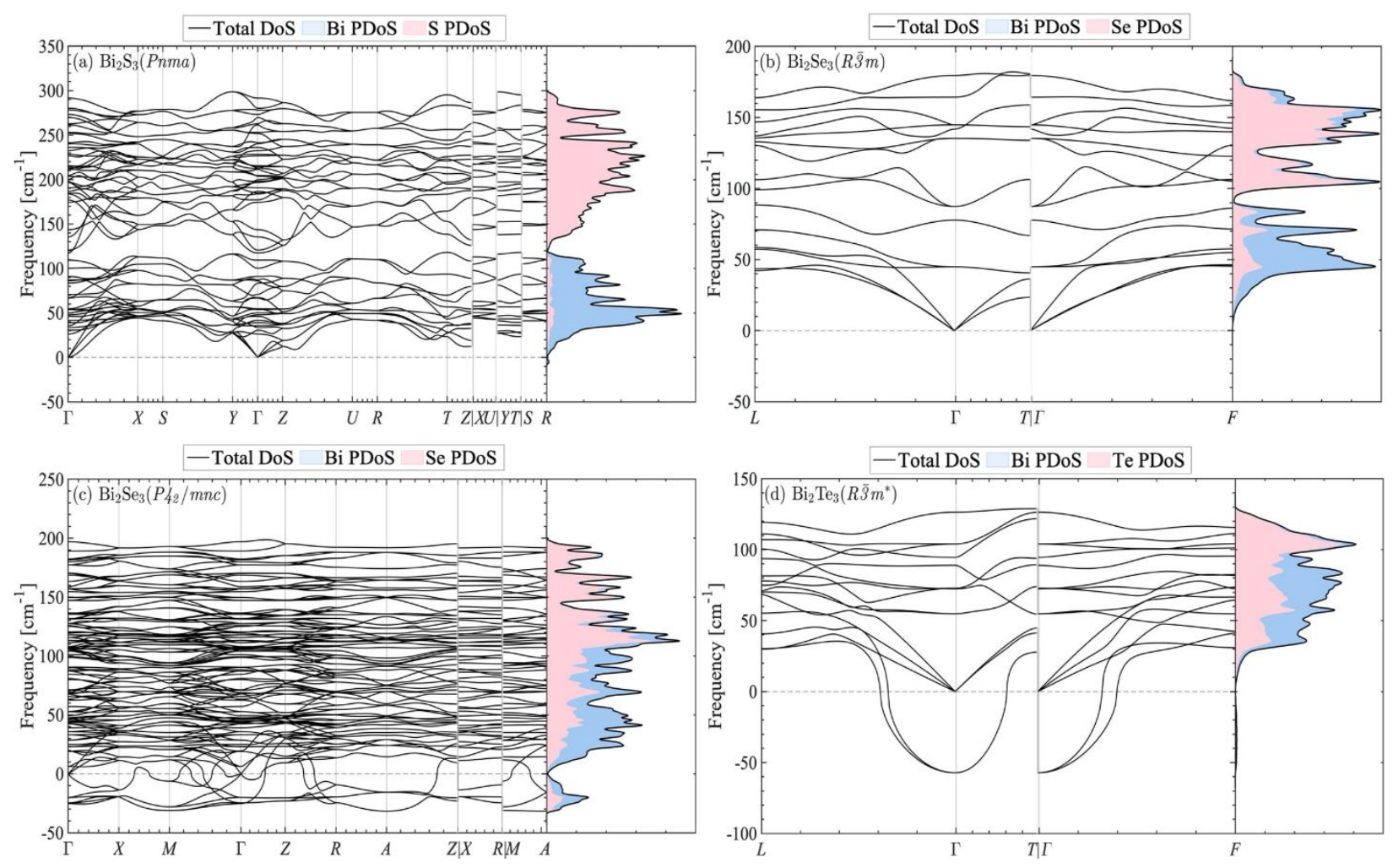

Figure 2 Representative phonon dispersion and density of states (DoS) curves of the four bismuth chalcogenide phases considered in this work: Pnma $\mathrm{Bi}_{2} \mathrm{~S}_{3}$ (a), $R \overline{3} m \mathrm{Bi}_{2} \mathrm{Se}_{3}$ (b), $P 4_{2} / n m c \mathrm{Bi}_{2} \mathrm{Se}_{3}$ (c) and $R \overline{3} m^{*} \mathrm{Bi}_{2} \mathrm{Te}_{3}(\mathrm{~d})$. The projections of the DoS onto the bismuth and chalcogen atoms are shown in blue and pink, respectively, as stacked area plots. Equivalent plots for $P n m a \mathrm{Bi}_{2} \mathrm{Se}_{3} / \mathrm{Bi}_{2} \mathrm{Te}_{3}$ and $R \overline{3} m \mathrm{Bi}_{2} \mathrm{~S}_{3} / \mathrm{Bi}_{2} \mathrm{Te}_{3}$ are given in the Supporting Information.

where $A^{\mathrm{vib}}=U^{\mathrm{vib}}(T)-T S^{\mathrm{vib}}(T)$ is the contribution to the free energy from the phonon modes and $U^{\mathrm{vib}}$ and $S^{\mathrm{vib}}$ are the phonon internal energy and entropy respectively. When two phases are close in energy, differences in phonon spectra and $A^{\mathrm{vib}}$ can lead to qualitative changes in stability at $0 \mathrm{~K}$ and/or to temperature-induced phase transitions. ${ }^{67}$ 

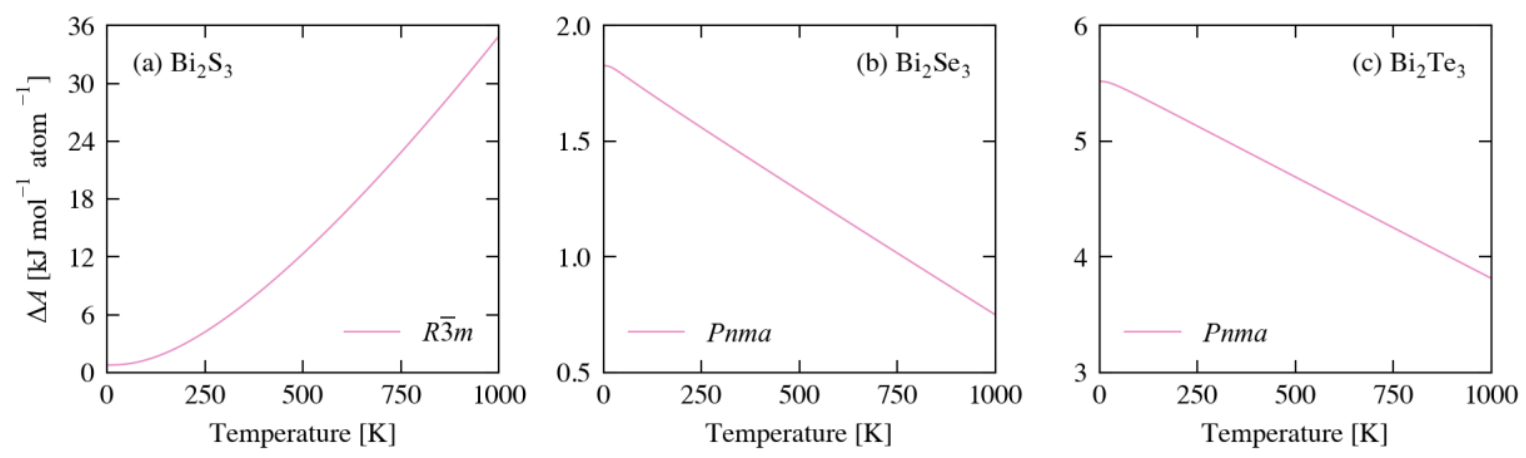

Figure 3 Constant-volume (Helmholtz) free-energy differences $\Delta A$ between the Pnma and $R \overline{3} m$ phases of $\mathrm{Bi}_{2} \mathrm{~S}_{3}$ (a), $\mathrm{Bi}_{2} \mathrm{Se}_{3}$ (b) and $\mathrm{Bi}_{2} \mathrm{Te}_{3}$ (c) as a function of temperature. In each subplot the $\Delta A$ are shown relative to the phase with the lowest energy at $T=0 \mathrm{~K}$, which is the Pnma phase for $\mathrm{Bi}_{2} \mathrm{~S}_{3}$ and the $R \overline{3} m$ phase for $\mathrm{Bi}_{2} \mathrm{Se}_{3}$ and $\mathrm{Bi}_{2} \mathrm{Te}_{3}$ (c.f. Table 3).

Figure 3 compares the energy differences $\Delta A$ between the Pnma and $R \overline{3} m$ phases of $\mathrm{Bi}_{2} \mathrm{~S}_{3}$, $\mathrm{Bi}_{2} \mathrm{Se}_{3}$ and $\mathrm{Bi}_{2} \mathrm{Te}_{3}$. In all three, we find that the Pnma phase is lowered in energy relative to the competing $R \overline{3} m$ phase with increasing temperature, indicating that the former is stabilized by differences in the vibrational free energy. For $\mathrm{Bi}_{2} \mathrm{~S}_{3}$, this means that the energy difference between the ground-state Pnma and the $R \overline{3} m$ phase increases sharply with temperature. $\mathrm{For} \mathrm{Bi}_{2} \mathrm{Se}_{3}$ and $\mathrm{Bi}_{2} \mathrm{Te}_{3}$, on the other hand, the free-energy differences between the Pnma and $R \overline{3} m$ phases fall with temperature. For $\mathrm{Bi}_{2} \mathrm{Se}_{3}$, the $\Delta A$ falls from $\sim 1.8 \mathrm{~kJ} \mathrm{~mol}^{-1}$ atom ${ }^{-1}$ at $0 \mathrm{~K}$ to $0.75 \mathrm{~kJ} \mathrm{~mol}^{-1}$ atom ${ }^{-1}$ at $1000 \mathrm{~K}$, whereas for $\mathrm{Bi}_{2} \mathrm{Te}_{3}$ the differences range from $\sim 5.5 \mathrm{~kJ} \mathrm{~mol}^{-1}$ atom ${ }^{-1}$ at $0 \mathrm{~K}$ to $\sim 3.9 \mathrm{~kJ} \mathrm{~mol}^{-}$ ${ }^{1}$ atom ${ }^{-1}$ at $1000 \mathrm{~K}$. (The melting temperatures of $\mathrm{Bi}_{2} \mathrm{Se}_{3}$ and $\mathrm{Bi}_{2} \mathrm{Te}_{3}$ are around 983 and $858 \mathrm{~K}$, respectively.) While this analysis further highlights the chemical trend in the energetic preference for the $R \overline{3} m$ structure in the heavier chalcogenides, the calculations do not suggest any thermal 
phase transitions and predict the same qualitative stability ordering as the lattice energies (c.f.

Table 3). Nonetheless, the Pnma phase of $\mathrm{Bi}_{2} \mathrm{Se}_{3}$ is close in energy to the $R \overline{3} m$ phase, and it may therefore be possible to stabilize it by alloying with $\mathrm{Bi}_{2} \mathrm{~S}_{3}$ or by epitaxial growth on a suitable substrate. We return to the former point in a following subsection.

Lattice Thermal Conductivity of Pnma and $R \overline{3} m \mathrm{Bi}_{2} \mathrm{~S}_{3}, \mathrm{Bi}_{2} \mathrm{Se}_{3}$ and $\mathrm{Bi}_{2} \mathrm{Te}_{3}$. Using the single-mode relaxation-time approximation, the lattice thermal conductivity $\kappa_{\text {latt }}$ is computed as a sum over contributions $\boldsymbol{\kappa}_{\lambda}$ from individual phonon modes $\lambda$ according to:

$$
\boldsymbol{\kappa}_{\text {latt }}(T)=\frac{1}{N} \sum_{\lambda} \boldsymbol{\kappa}_{\lambda}(T)=\frac{1}{N V_{0}} \sum_{\lambda} C_{\lambda}(T) \boldsymbol{v}_{\lambda} \otimes \boldsymbol{v}_{\lambda} \tau_{\lambda}(T)
$$

where $C_{\lambda}$ are the modal heat capacities, $\boldsymbol{v}_{\lambda}$ are the model group velocities, $\tau_{\lambda}$ are the phonon lifetimes, $N$ is the number of wavevectors included in the summation, and $V_{0}$ is the unit cell volume. $C_{\lambda}$ and $\boldsymbol{v}_{\lambda}$ are calculated within the harmonic approximation as:

$$
\begin{gathered}
C_{\lambda}(T)=k_{\mathrm{B}}\left(\frac{\hbar \omega_{\lambda}}{k_{\mathrm{B}} T}\right)^{2} \frac{\exp \left[\hbar \omega_{\lambda} / k_{\mathrm{B}} T\right]}{\left(\exp \left[\hbar \omega_{\lambda} / k_{\mathrm{B}} T\right]-1\right)^{2}} \\
\boldsymbol{v}_{\lambda}=\frac{\partial \omega_{\lambda}}{\partial \mathbf{q}_{\lambda}}
\end{gathered}
$$


Where $\omega_{\lambda}$ are the (angular) phonon frequencies and $k_{\mathrm{B}}$ is the Boltzmann constant. The $\tau_{\lambda}$ are determined as the inverse of the phonon linewidths $\Gamma_{\lambda}$ according to:

$$
\tau_{\lambda}(T)=\frac{1}{2 \Gamma_{\lambda}(T)}
$$

The $\Gamma_{\lambda}$ are computed as the imaginary part of the phonon self-energies from a perturbative expression using the harmonic frequencies and eigenvectors and the third-order force constants full details of this method are given in Ref. ${ }^{28}$.

Figure 4 compares the calculated $\kappa_{\text {latt }}$ of the Pnma and $R \overline{3} m$ phases of $\mathrm{Bi}_{2} \mathrm{~S}_{3}, \mathrm{Bi}_{2} \mathrm{Se}_{3}$ and $\mathrm{Bi}_{2} \mathrm{Te}_{3}$ as a function of temperature. Table 4 compares the room-temperature ( $\left.300 \mathrm{~K}\right)$ values of the three principal diagonal components of the $\boldsymbol{\kappa}_{\text {latt }}$ tensor, $\kappa_{x x}, \kappa_{y y}$ and $\kappa_{z z}$, together with the isotropic average $\kappa_{\text {ave }}=\frac{1}{3} \operatorname{Tr}\left[\kappa_{\text {latt }}\right]=\frac{1}{3}\left(\kappa_{x x}+\kappa_{y y}+\kappa_{z z}\right)$. We calculate a $\kappa_{\text {ave }}$ of 0.9 for Pnma $\mathrm{Bi}_{2} \mathrm{~S}_{3}$, which compares favorably to the experimental value of $0.87 \mathrm{~W} \mathrm{~m}^{-1} \mathrm{~K}^{-1}{ }^{68}$ Our predicted $\kappa_{\text {ave }}$ for $R \overline{3} m \mathrm{Bi}_{2} \mathrm{Se}_{3}$ and $\mathrm{Bi}_{2} \mathrm{Te}_{3}$ are 1.82 and $0.88 \mathrm{~W} \mathrm{~m}^{-1} \mathrm{~K}^{-1}$. The value for $\mathrm{Bi}_{2} \mathrm{Se}_{3}$ again compares favorably to the measured value of $1.7 \mathrm{~W} \mathrm{~m}^{-1} \mathrm{~K}^{-1}$ quoted in Ref. 15 . On the other hand, the latter significantly underestimates the values of 1.28 and $1.37 \mathrm{~W} \mathrm{~m}^{-1} \mathrm{~K}^{-1}$ measured for $\mathrm{Bi}_{2} \mathrm{Te}_{3} \cdot{ }^{15,69} \mathrm{The}$ reasons for this discrepancy are not clear. 


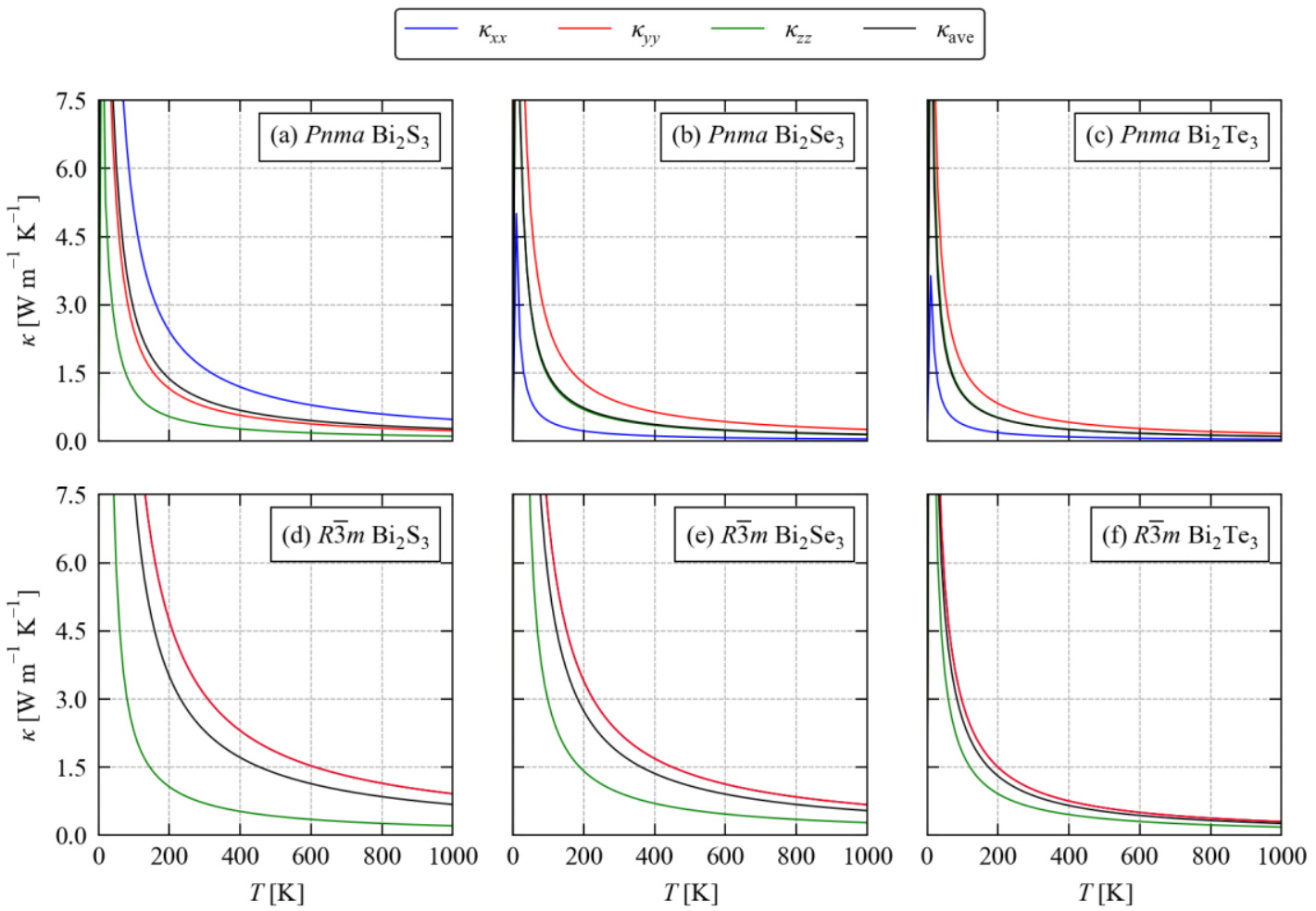

Figure 4 Calculated lattice thermal conductivity of the Pnma (a-c) and $R \overline{3} m$ (d-f) phases of $\mathrm{Bi}_{2} \mathrm{~S}_{3}$ (a, d), $\mathrm{Bi}_{2} \mathrm{Se}_{3}(\mathrm{~b}, \mathrm{e})$ and $\mathrm{Bi}_{2} \mathrm{Te}_{3}(\mathrm{c}, \mathrm{f})$ as a function of temperature. Each plot shows the principal diagonal elements of the $\kappa_{\text {latt }}$ tensor, $\kappa_{x x}, \kappa_{y y}$ and $\kappa_{z z}$, together with the isotropic average $\kappa_{\text {ave }}=$ $\left(\kappa_{x x}+\kappa_{y y}+\kappa_{z z}\right) / 3$. Note that the ordering of the axes is different between Pnma $\mathrm{Bi}_{2} \mathrm{~S}_{3}$ and $\mathrm{Bi}_{2} \mathrm{Se}_{3} / \mathrm{Bi}_{2} \mathrm{Te}_{3}$ (c.f. Table 2), and that in the $R \overline{3} m$ phases $\kappa_{x x}=\kappa_{y y}$ by symmetry.

Several trends are apparent from the calculations. For a given structure type (i.e. Pnma or $R \overline{3} m)$, the $\kappa_{\text {latt }}$ decreases with increasing chalcogen mass as expected. More interestingly, for a given chalcogen the average $\kappa_{\text {latt }}$ of the Pnma structure is consistently smaller than that of the $R \overline{3} m$ phase, by $60-70 \%$. 
Table 4 Room-temperature $\left(300 \mathrm{~K}\right.$ ) lattice thermal conductivity $\kappa_{\text {latt }}$ of $\mathrm{Bi}_{2} \mathrm{~S}_{3}, \mathrm{Bi}_{2} \mathrm{Se}_{3}$ and $\mathrm{Bi}_{2} \mathrm{Te}_{3}$ in the Pnma and $R \overline{3} m$ phases; the $\kappa_{x x}, \kappa_{y y}$ and $\kappa_{z z}$ components of the $\kappa_{\text {latt }}$ tensor are given together with the average $\kappa_{\text {ave }}=\left(\kappa_{x x}+\kappa_{y y}+\kappa_{z z}\right) / 3$, and experimental measurements are given for comparison where available.

\begin{tabular}{|c|c|c|c|c|c|}
\hline & \multicolumn{5}{|c|}{$\kappa_{\text {latt }}\left[\mathrm{W} \mathrm{m}^{-1} \mathrm{~K}^{-1}\right]$} \\
\hline & \multicolumn{4}{|c|}{ Calc. (this work) } & \multirow[b]{2}{*}{ Expt. } \\
\hline & $\kappa_{x x}$ & $\kappa_{y y}$ & $\kappa_{z z}$ & $\kappa_{\text {ave }}$ & \\
\hline $\mathrm{Bi}_{2} \mathrm{~S}_{3}(P n m a)$ & 1.593 & 0.760 & 0.357 & 0.903 & $\begin{array}{c}0.87 \\
(T=325 \mathrm{~K})^{68}\end{array}$ \\
\hline $\mathrm{Bi}_{2} \mathrm{~S}_{3}(R \overline{3} m)$ & 3.091 & 3.091 & 0.705 & 2.296 & - \\
\hline $\mathrm{Bi}_{2} \mathrm{Se}_{3}(P n m a)$ & 0.147 & 0.850 & 0.462 & 0.487 & - \\
\hline $\mathrm{Bi}_{2} \mathrm{Se}_{3}(R \overline{3} m)$ & 2.260 & 2.260 & 0.939 & 1.819 & $1.70^{15}$ \\
\hline $\mathrm{Bi}_{2} \mathrm{Te}_{3}(P n m a)$ & 0.123 & 0.551 & 0.346 & 0.340 & - \\
\hline $\mathrm{Bi}_{2} \mathrm{Te}_{3}(R \overline{3} m)$ & 1.007 & 1.007 & 0.611 & 0.875 & $\begin{array}{l}1.28^{69} \\
1.37^{15}\end{array}$ \\
\hline
\end{tabular}

This is most noteworthy for $\mathrm{Bi}_{2} \mathrm{Se}_{3}$, which has been reported in both structure types and for which our calculations predict the Pnma and $R \overline{3} m$ phases to be energetically competitive. Finally, we find that for both structure types the $\kappa_{\text {latt }}$ is predicted to be anisotropic. In the Pnma phases, the conductivity is largest along the short axes (the $a$ axis for $\mathrm{Bi}_{2} \mathrm{~S}_{3}$ and the $b$ axes for $\mathrm{Bi}_{2} \mathrm{Se}_{3}$ and $\mathrm{Bi}_{2} \mathrm{Te}_{3}$; c.f. Table 3 ). The $\kappa_{\text {latt }}$ is consistently around $80 \%$ smaller along one of the two orthogonal axes, and is 52,46 and $37 \%$ smaller along the other axis in $\mathrm{Bi}_{2} \mathrm{~S}_{3}, \mathrm{Bi}_{2} \mathrm{Se}_{3}$ and $\mathrm{Bi}_{2} \mathrm{Te}_{3}$ respectively. The anisotropy in the thermal transport decreases for the heavier chalcogens, but all three Pnma systems retain distinct "easy" and "hard" axes. For the $R \overline{3} m$, the transport along the $x$ and $y$ directions corresponds to transport in the $a b$ plane, and is $4.4,2.4$ and $1.6 \times$ larger than the transport in the cross-plane $z$ direction ( $c$ axis) in $\mathrm{Bi}_{2} \mathrm{~S}_{3}, \mathrm{Bi}_{2} \mathrm{Se}_{3}$ and $\mathrm{Bi}_{2} \mathrm{Te}_{3}$ respectively. As for the Pnma systems, the anisotropy therefore decreases with increasing chalcogen mass, but even for $\mathrm{Bi}_{2} \mathrm{Te}_{3}$ the cross-plane transport is $40 \%$ smaller than the in-plane transport. 
To better understand the origin of the trends in the $\kappa_{\text {latt }}$, we use the constant relaxationtime approximation (CRTA) model developed in our previous work. ${ }^{46,70}$ We write the $\boldsymbol{\kappa}_{\text {latt }}$ as the product of a temperature-dependent harmonic function $\boldsymbol{\kappa} / \tau^{\mathrm{CRTA}}$ and weighted average lifetime $\tau^{\text {CRTA }}$ defined according to:

$$
\boldsymbol{\kappa}_{\text {latt }}(T)=\tau^{\mathrm{CRTA}} \times \frac{1}{N} \sum_{\lambda} \frac{\boldsymbol{\kappa}_{\lambda}(T)}{\tau_{\lambda}(T)}=\tau^{\mathrm{CRTA}} \times \frac{1}{N V_{0}} \sum_{\lambda} C_{\lambda}(T) \boldsymbol{v}_{\lambda} \otimes \boldsymbol{v}_{\lambda}
$$

where the terms are defined as in Eq. 3 above. As for $\boldsymbol{\kappa}_{\text {latt }}$ the $\boldsymbol{\kappa} / \tau^{\mathrm{CRTA}}$ are tensors, and the $\tau^{\mathrm{CRTA}}$ may be taken as a scalar, like the $\tau_{\lambda}$, or as a tensor to provide some flexibility to account for anisotropy in the averaged mode lifetimes. ${ }^{46,70}$

Table 5 Constant relaxation-time approximation (CRTA) analysis of the room-temperature averaged thermal conductivities $\kappa_{\text {ave }}$ of $\mathrm{Bi}_{2} \mathrm{~S}_{3}, \mathrm{Bi}_{2} \mathrm{Se}_{3}$ and $\mathrm{Bi}_{2} \mathrm{Te}_{3}$ in the Pnma and $R \overline{3} m$ phases according to Eq. 7 in the text.

\begin{tabular}{lccc}
\hline & $\begin{array}{c}\kappa_{\text {ave }} \\
{\left[\mathrm{W} \mathrm{m}^{-1} \mathrm{~K}^{-1}\right]}\end{array}$ & $\begin{array}{c}\kappa / \tau^{\mathrm{CRTA}} \\
{\left[\mathrm{W} \mathrm{m} \mathrm{K}^{-1} \mathrm{ps}^{-1}\right]}\end{array}$ & $\begin{array}{c}\tau^{\mathrm{CRTA}} \\
{[\mathrm{ps}]}\end{array}$ \\
\hline $\mathrm{Bi}_{2} \mathrm{~S}_{3}($ Pnma $)$ & 0.903 & 0.422 & 2.14 \\
\hline $\mathrm{Bi}_{2} \mathrm{~S}_{3}(R \overline{3} m)$ & 2.296 & 0.754 & 3.04 \\
\hline $\mathrm{Bi}_{2} \mathrm{Se}_{3}(P n m a)$ & 0.487 & 0.198 & 2.45 \\
\hline $\mathrm{Bi}_{2} \mathrm{Se}_{3}(R \overline{3} m)$ & 1.819 & 0.293 & 6.20 \\
\hline $\mathrm{Bi}_{2} \mathrm{Te}_{3}($ Pnma $)$ & 0.340 & 0.103 & 3.31 \\
\hline $\mathrm{Bi}_{2} \mathrm{Te}_{3}(R \overline{3} m)$ & 0.875 & 0.199 & 4.41 \\
\hline
\end{tabular}



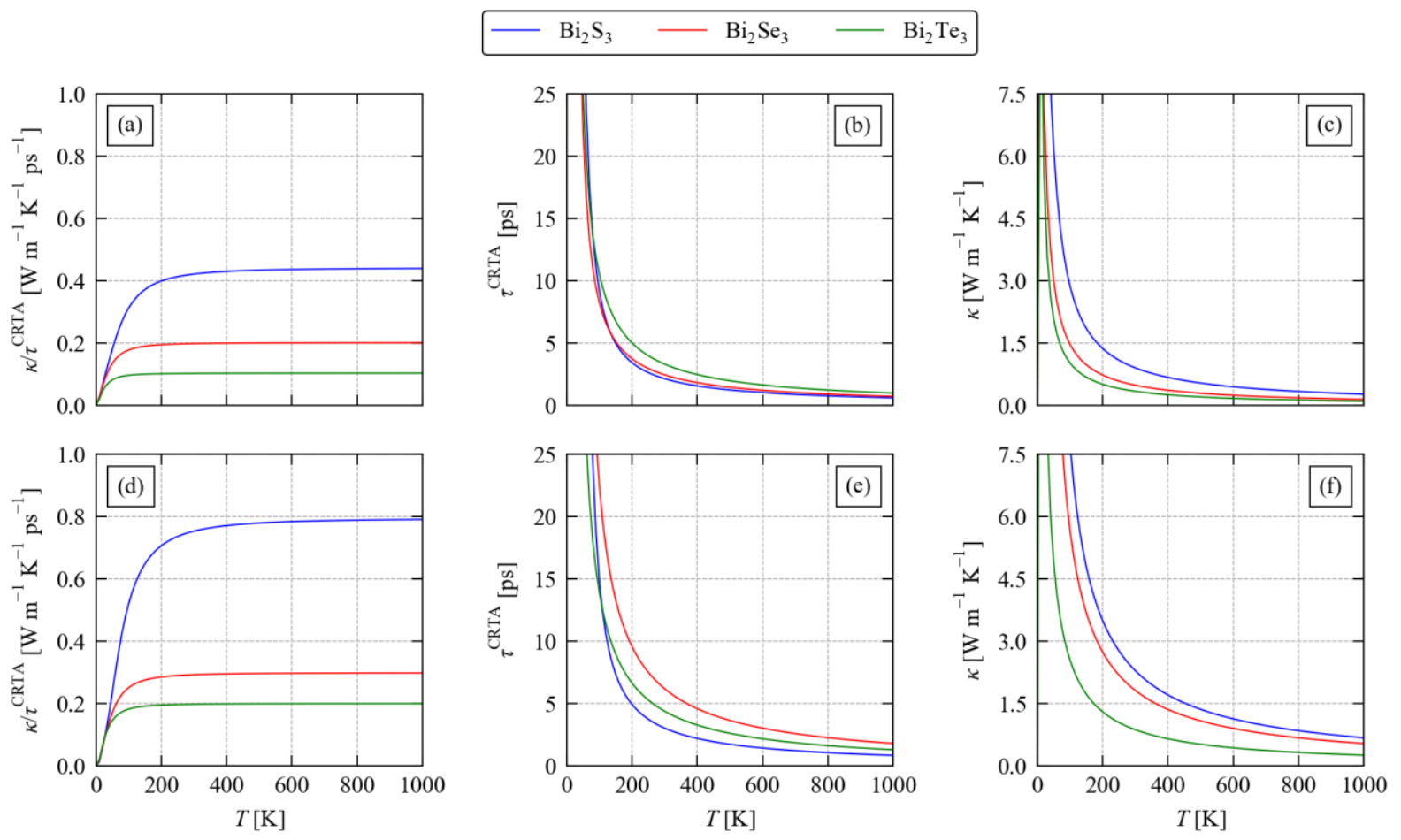

Figure 5 Analysis of the averaged lattice thermal conductivity $\kappa_{\text {ave }}=\left(\kappa_{x x}+\kappa_{y y}+\kappa_{z z}\right) / 3$ of $\mathrm{Bi}_{2} \mathrm{~S}_{3}, \mathrm{Bi}_{2} \mathrm{Se}_{3}$ and $\mathrm{Bi}_{2} \mathrm{Te}_{3}$ in the Pnma (a-c) and $R \overline{3} m$ (d-f) phases using the constant-relaxation time approximation (CRTA) model defined in Eq. 7 in the text. (a) and (d) compare the temperature dependence of the harmonic term $\kappa / \tau^{\mathrm{CRTA}}$ of the three chalcogenides in both phases, (b) and (e) compare the averaged lifetimes $\tau^{\mathrm{CRTA}}$, and (c) and (f) compare the $\kappa_{\text {ave }}$.

Figure 5 compares the $\kappa_{\text {ave }}$ and corresponding $\kappa / \tau^{\mathrm{CRTA}}$ and $\tau^{\mathrm{CRTA}}$ for the two phases of the three chalcogenides as a function of temperature, and room-temperature values of these quantities are listed in Table 5. Based on this analysis, the lower room-temperature $\kappa_{\text {latt }}$ of the Pnma phases can be explained by a 30-50\% reduction in the harmonic term and a 25-60\% reduction in the weighted average lifetime. This implies that the Pnma structure lends itself to both 
weaker chemical bonding and stronger phonon anharmonicity. As expected, for a given structure type the harmonic term decreases with increasing chalcogen mass, falling by 53 and $76 \%$ in Pnma $\mathrm{Bi}_{2} \mathrm{Se}_{3}$ and $\mathrm{Bi}_{2} \mathrm{Te}_{3}$ relative to $\mathrm{Bi}_{2} \mathrm{~S}_{3}$, and by 61 and $74 \%$ in $R \overline{3} m \mathrm{Bi}_{2} \mathrm{Se}_{3}$ and $\mathrm{Bi}_{2} \mathrm{Te}_{3}$ relative to the corresponding $\mathrm{Bi}_{2} \mathrm{~S}_{3}$ phase. Interestingly, for the Pnma phases the lifetimes increase for the heavier chalcogens, from 2.14 ps in Pnma $\mathrm{Bi}_{2} \mathrm{~S}_{3}$ to 2.45 and 3.31 ps in $\mathrm{Bi}_{2} \mathrm{Se}_{3}$ and $\mathrm{Bi}_{2} \mathrm{Te}_{3}$. For the $R \overline{3} m$ phases the lifetimes increase by a factor of two from $\mathrm{Bi}_{2} \mathrm{~S}_{3}$ to $\mathrm{Bi}_{2} \mathrm{Se}_{3}$, but are reduced by $\sim 25 \%$ in $\mathrm{Bi}_{2} \mathrm{Te}_{3}$ compared to $\mathrm{Bi}_{2} \mathrm{Se}_{3}$. An increase in lifetime counteracting a reduced harmonic term was also observed when comparing the thermal conductivity of $\mathrm{SnS}$ and $\mathrm{SnSe}^{46}$

This method of analyzing the $\boldsymbol{\kappa}_{\text {latt }}$ and comparing between materials is relatively new, but we have previously performed similar calculations on $\mathrm{SnS} / \mathrm{SnSe}$ and $\mathrm{CoSb}_{3},{ }^{46,70}$ all of which are widely-studied thermoelectric materials. For $\mathrm{SnSe}$, we calculated a room-temperature $\kappa_{\text {ave }}$ of 1.58 $\mathrm{W} \mathrm{m}{ }^{-1} \mathrm{~K}^{-1}$ together with a $\kappa / \tau^{\mathrm{CRTA}}$ of $0.372 \mathrm{~W} \mathrm{~m}^{-1} \mathrm{~K}^{-1} \mathrm{ps}^{-1}$ and a $\tau^{\mathrm{CRTA}}$ of $4.23 \mathrm{ps}^{46}$ The $\kappa_{\text {ave }}$ falls between the Pnma and $R \overline{3} m$ phases of $\mathrm{Bi}_{2} \mathrm{Se}_{3}$. The harmonic term is larger than in both materials, and the higher $\kappa_{\text {ave }}$ of $R \overline{3} m \mathrm{Bi}_{2} \mathrm{Se}_{3}$ is thus due to its longer average lifetimes while the lower thermal conductivity of Pnma $\mathrm{Bi}_{2} \mathrm{Se}_{3}$ is due to both the smaller harmonic term and shorter $\tau^{\mathrm{CRTA}}$. For $\mathrm{CoSb}_{3}$, we obtained $\kappa_{\text {ave }}=9.98 \mathrm{~W} \mathrm{~m}^{-1} \mathrm{~K}^{-1}$ with $\kappa / \tau^{\mathrm{CRTA}}=0.273 \mathrm{~W} \mathrm{~m}^{-1} \mathrm{~K}^{-1} \mathrm{ps}^{-1}$ and $\tau^{\mathrm{CRTA}}=36.6 \mathrm{ps}$. The harmonic term is smaller than in both sulfides, comparable to the $R \overline{3} m$ phase of $\mathrm{Bi}_{2} \mathrm{Se}_{3}$, and larger than both tellurides. However, the lifetime is much larger than all six chalcogenide materials investigated here, by a factor of 6-17 $\times$, and this is the main reason for its much larger thermal conductivity. 
Thermodynamics of Binary Solid Solutions. Based on the relative energies of the Pnma and $R \overline{3} m$ phases of $\mathrm{Bi}_{2} \mathrm{~S}_{2}, \mathrm{Bi}_{2} \mathrm{Se}_{3}$ and $\mathrm{Bi}_{2} \mathrm{Te}_{3}$ discussed above, we would expect $\mathrm{Bi}_{2}\left(\mathrm{Se}_{1-x} \mathrm{~S}_{x}\right)_{3}$ to potentially form in the Pnma or $R \overline{3} m$ structures, whereas we would only expect $\mathrm{Bi}_{2}\left(\mathrm{Se}_{1-x} \mathrm{Te}_{x}\right)_{3}$ to form in the $R \overline{3} m$ structure. This is largely borne out in experimental reports, although $\mathrm{Bi}_{2} \mathrm{Te}_{2} \mathrm{~S}$ has been reported in the $R \overline{3} m$ structure. ${ }^{19}$ We therefore investigated the mixing thermodynamics of these three alloy compositions following the approach adopted in our previous work on the $\operatorname{Sn}_{n}\left(\mathrm{~S}_{1}\right.$ $\left.{ }_{x} \mathrm{Se}_{x}\right)_{m}$ system. ${ }^{44,71}$

Systematically substituting the chalcogen atoms in the 20-atom Pnma $\mathrm{Bi}_{2} \mathrm{~S}_{3}$ primitive cell yields 1,072 unique structures in 13 compositions between the $\mathrm{Bi}_{2} \mathrm{~S}_{3}$ and $\mathrm{Bi}_{2} \mathrm{Se}_{3}$ endpoints, from which we analyzed every second composition (560 structures). Applying the same procedure to a $2 \times 2 \times 1$ supercell expansion of the $R \overline{3} m$ primitive cell yields 378 unique structures in 13 compositions between the $\mathrm{Bi}_{2} \mathrm{~S}_{3} / \mathrm{Bi}_{2} \mathrm{Se}_{3}$ and $\mathrm{Bi}_{2} \mathrm{Se}_{3} / \mathrm{Bi}_{2} \mathrm{Te}_{3}$ endpoints (i.e. 756 structures in total), from which we analyzed all 13 compositions.

For a given composition, the solid-solution model thus comprises $n_{x}$ symmetryinequivalent chalcogen arrangements with total energy $E_{n}$, which we equate to the lattice internal energy $U_{n}^{\text {latt }}$, and an associated degeneracy $g_{n}$. These are used to build the thermodynamic partition function $Z(x ; T)$ :

$$
Z(x ; T)=\sum_{n=1}^{n_{x}} g_{n} \exp \left[-U_{n}^{\mathrm{latt}} / k_{\mathrm{B}} T\right]
$$


The Helmholtz energy $A(x ; T)$ can be computed from the $Z(x ; T)$ using the bridge relation from statistical mechanics:

$$
A(x ; T)=-k_{\mathrm{B}} T \ln Z(x ; T)=\bar{U}^{\mathrm{latt}}(x ; T)-T S^{\mathrm{conf}}(x ; T)
$$

where $\bar{U}^{\text {latt }}(x ; T)$ is the thermodynamic average of the lattice internal energy and $S^{\operatorname{conf}}(x ; T)$ is the configurational entropy. One can then compute a mixing energy $A_{\operatorname{mix}}(x ; T)$ as:

$$
A_{\text {mix }}(x ; T)=A(x ; T)-[(1-x) A(x=0 ; T)+x A(x=1 ; T)]
$$

where $A(x=0 ; T)$ and $A(x=1 ; T)$ are the mixing Helmholtz energies of the endpoints which, given the $g_{n}$ are unity by construction, are equal to the corresponding $U_{n}^{\text {latt }}$.

Given a $Z(x ; T)$, the occurrence probabilities $P_{n}$ of the individual structures in each composition can be computed as:

$$
P_{n}(x ; T)=\frac{1}{Z(x ; T)} g_{n} \exp \left[-U_{n}^{\mathrm{latt}} / k_{\mathrm{B}} T\right]
$$

The $P_{n}$ can the be used to compute the thermodynamic average $\bar{X}$ of a physical property $X$ according to: 


$$
\bar{X}(x ; T)=\sum_{n=1}^{n_{x}} P_{n}(x ; T) \times X_{n}
$$

In particular, the $\bar{U}^{\text {latt }}$ in Eq. 9 can be computed as a weighted average of the $U_{n}^{\text {latt }}$, and subtracted from the Helmholtz energy to obtain the $-T S^{\text {conf }}$ term. One can then separate the mixing energies $A_{\text {mix }}$ into contributions from internal energy and entropy $\left(U_{\mathrm{mix}} /-T S_{\mathrm{mix}}\right)$. It is worth noting that the structure of the alloy is generally assumed to be set during its formation, so the averaged properties are predicted using $P_{n}$ computed for a set formation temperature $T_{\mathrm{F}} \cdot{ }^{71}$

Figure 6 shows the calculated mixing energies of the three alloys as a function of composition and for formation temperatures from $300 \mathrm{~K}$ to $1200 \mathrm{~K}$. For the Pnma $\mathrm{Bi}_{2}\left(\mathrm{Se}_{1-x} \mathrm{~S}_{x}\right)_{3}$ alloy, the profile is similar to what would be expected for an ideal solid solution where the mixing is driven by the configurational entropy. For the two $R \overline{3} m$ alloys, on the other hand, there is a notable skew in the mixing profiles, particularly at low temperature, towards $x=1 / 3$ for the $\mathrm{Bi}_{2}\left(\mathrm{Se}_{1-x} \mathrm{~S}_{x}\right)_{3}$ alloy (i.e. $\left.\mathrm{Bi}_{2} \mathrm{SSe}_{2}\right)$ and toward $x=2 / 3$ for the $\mathrm{Bi}_{2}\left(\mathrm{Se}_{1-x} \mathrm{Te}_{x}\right)_{3}$ alloy (i.e. $\left.\mathrm{Bi}_{2} \mathrm{SeTe}_{2}\right)$. Consideration of the contributions to the $A_{\text {mix }}$ from the $U_{\text {mix }}$ and $-T S_{\text {mix }}$ terms (Figures S9-S11) indicates that these compositions are associated with a significantly reduced (i.e. more negative/favorable) enthalpy and a decrease in entropy, both of which are again most pronounced at lower formation temperatures.

Taken together, these results indicate a strong energetic preference for particular configurations. This can be understood by the fact that there are two distinct chalcogen environments in the $R \overline{3} m$ structure, which we refer to as $\mathrm{Chal}^{(1)}$ and $\mathrm{Chal}^{(2)}$. 

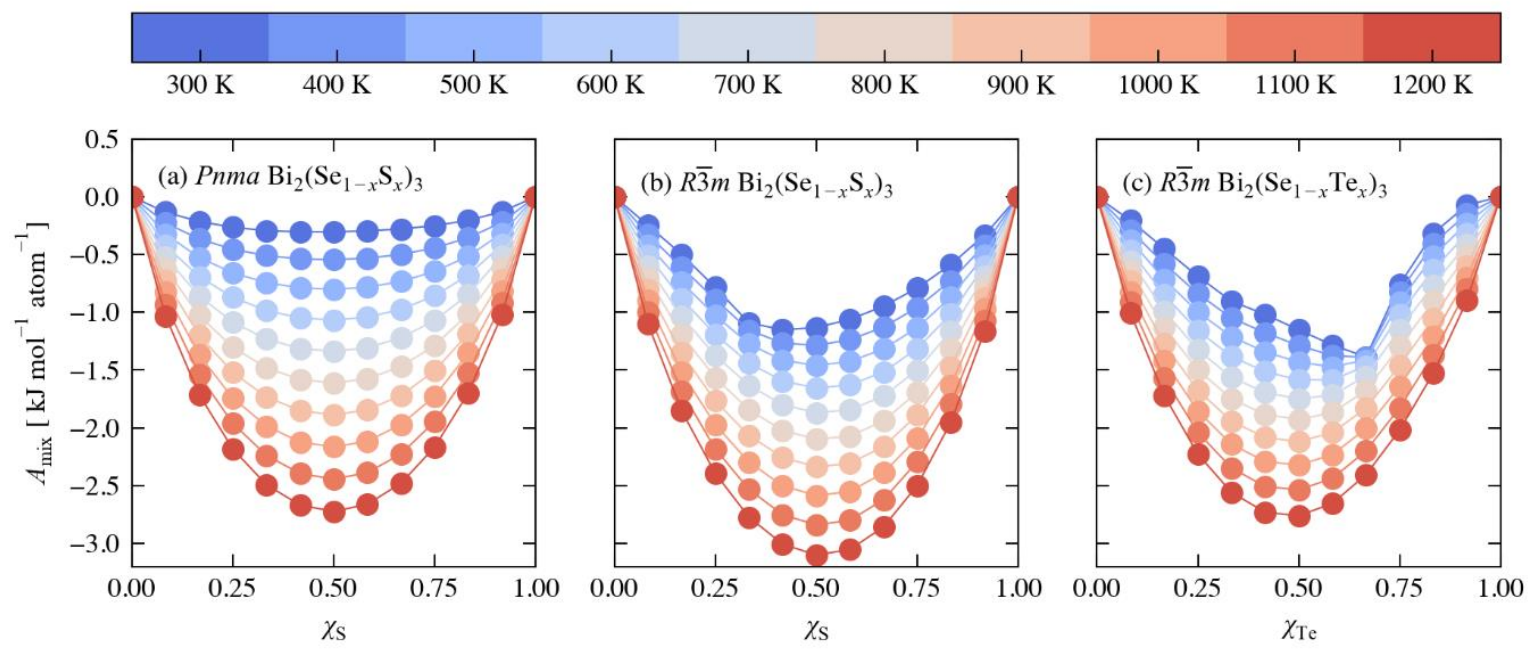

Figure 6 Calculated free energies of mixing $A_{\text {mix }}$ as a function of composition $x$ for $P n m a \mathrm{Bi}_{2}\left(\mathrm{Se}_{1}\right.$ $\left.{ }_{x} \mathrm{~S}_{x}\right)_{3}$ (a), $R \overline{3} m \mathrm{Bi}_{2}\left(\mathrm{Se}_{1-x} \mathrm{~S}_{x}\right)_{3}$ (b), and $R \overline{3} m \mathrm{Bi}_{2}\left(\mathrm{Se}_{1-x} \mathrm{Te}_{x}\right)_{3}$ (c). Each plot compares mixing profiles computed for alloy formation temperatures $T_{\mathrm{F}}$ from $300 \mathrm{~K}$ (blue) to $1200 \mathrm{~K}$ (red).

The single Chal ${ }^{(1)}$ position is in the interior of the layers, where the chalcogen atom forms three short and three long bonds to six Bi atoms in a distorted octahedral environment. The two Chal ${ }^{(2)}$ positions are at the edges of the quintuple layers, where the chalcogens form three covalent bonds to $\mathrm{Bi}$ atoms and project a lone pair of electrons into the interlayer spacing. It is thus more favorable for smaller and more electronegative anions to occupy the $\mathrm{Chal}^{(1)}$ sites, which benefit from stronger electrostatic interactions with the Bi cations, and for larger and more polarizable anions to occupy the $\mathrm{Chal}^{(2)}$ sites, which benefit from more space to accommodate sterically-depending lone pairs. Occupying the $\mathrm{Chal}^{(2)}$ sites with more polarizable anions may also facilitate stronger interlayer dispersion forces. In the $\mathrm{Bi}_{2}\left(\mathrm{Se}_{1-x} \mathrm{~S}_{x}\right)_{3}$ alloy, the preference would be to have $\mathrm{S}$ in the $\mathrm{Chal}^{(1)}$ sites and $\mathrm{Se}$ in the $\mathrm{Chal}^{(2)}$ sites, giving the favored $x=1 / 3$ stoichiometry. In $\mathrm{Bi}_{2}\left(\mathrm{Se}_{1-x} \mathrm{Te}_{x}\right)_{3}$, the 
preference would be for $\mathrm{Se}$ in the $\mathrm{Chal}^{(1)}$ sites and Te in the $\mathrm{Chal}^{(2)}$ sites, favoring the $x=2 / 3$ stoichiometry. That the favored compositions appear to have a stronger influence on the thermodynamics of the $\mathrm{Bi}_{2}\left(\mathrm{Se}_{1-x} \mathrm{Te}_{x}\right)_{3}$ alloy may be explained by the larger electronegativity difference between Se and Te and the fact that Te is the largest and most polarizable of the three chalcogens. We also note that this structural preference argument would explain why $\mathrm{Bi}_{2} \mathrm{Te}_{2} \mathrm{~S}$ is stable in the $R \overline{3} m$ phase. ${ }^{19}$

As expected following these arguments, the distribution of $P_{n}$ for structures in the $R \overline{3} m$ alloy models, particularly $\mathrm{Bi}_{2}\left(\mathrm{Se}_{1-x} \mathrm{Te}_{x}\right)_{3}$, are heavily skewed towards particular chalcogen arrangements in the alloy supercells (Figure 7). For $\mathrm{Bi}_{2} \mathrm{Se}_{2} \mathrm{~S}$ and $\mathrm{Bi}_{2} \mathrm{SeTe}_{2}$ at a formation temperature $T_{\mathrm{F}}=800 \mathrm{~K}$, the most probable chalcogen arrangements have $P_{n}=0.19$ and 0.42 , respectively, and the three structures (of 21) with the largest $P_{n}$ collectively account for 41 and 76 $\%$ of the cumulative probability. Even for the $x=1 / 2$ solid solution of $\mathrm{Bi}_{2}\left(\mathrm{Se}_{1-x} \mathrm{Te}_{x}\right)_{3}$, where the number of configurations and hence the configurational entropy are a maximum, the most probable four structures with the largest $P_{n}(4.8 \%$ of the total $)$ account for $68 \%$ of the cumulative probability. This is in stark contrast to the Pnma $\mathrm{Bi}_{2}\left(\mathrm{Se}_{1-x} \mathrm{~S}_{x}\right)_{3}$ alloy, where for the $x=1 / 3,1 / 2$ and 2/3 compositions the difference between the smallest and largest $P_{n}$ are $6.3 \times 10^{-2}, 1.9 \times 10^{-2}$ and $5.1 \times 10^{-2}$, respectively, indicating a much more homogenous distribution of configurations. It is interesting to note, however, that the calculations do predict a distribution over chalcogen arrangements in both $\mathrm{Bi}_{2} \mathrm{Se}_{2} \mathrm{~S}$ and $\mathrm{Bi}_{2} \mathrm{SeTe}_{2}$, at least for high-temperature syntheses, which suggests these "line" compounds might contain significant concentrations of defects where chalcogen atoms swap from their ideal crystallographic sites (i.e. antisite defects). 


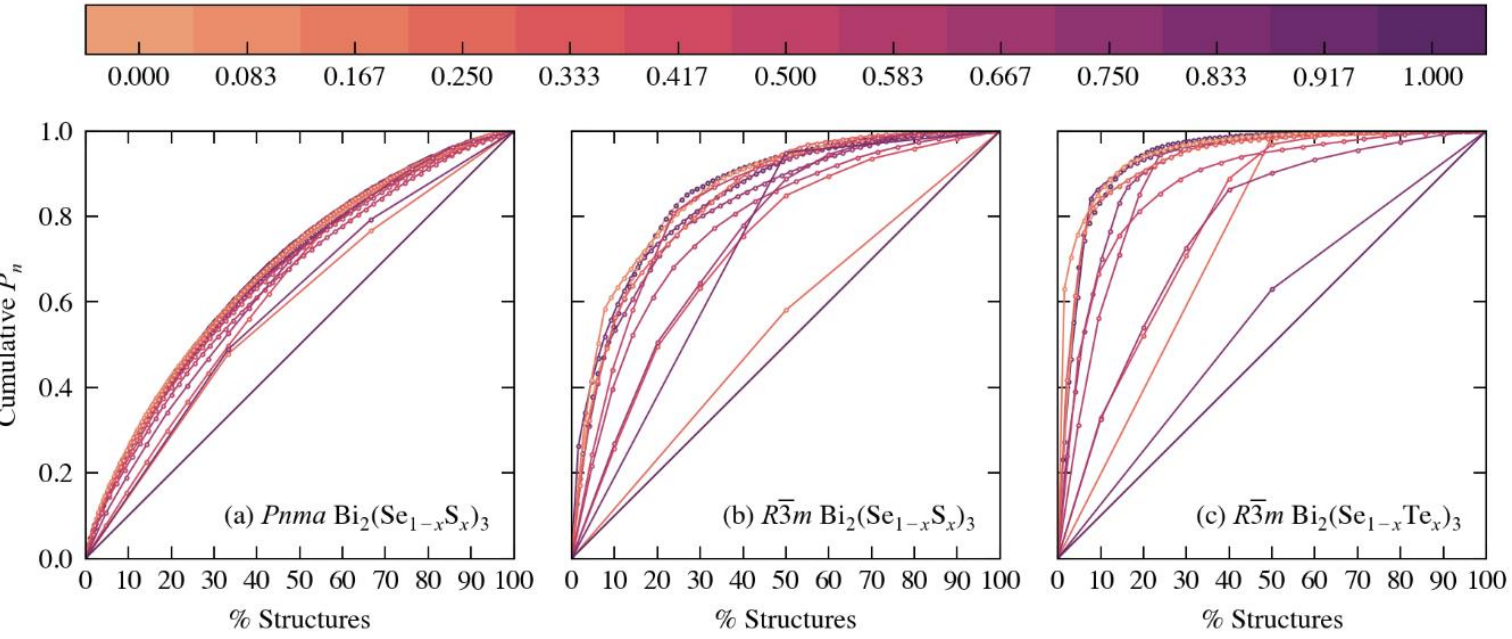

Figure 7 Distribution of occurrence probabilities $P_{n}$ over the structures in each composition of Pnma $\mathrm{Bi}_{2}\left(\mathrm{Se}_{1-x} \mathrm{~S}_{x}\right)_{3}$ (a), $R \overline{3} m \mathrm{Bi}_{2}\left(\mathrm{Se}_{1-x} \mathrm{~S}_{x}\right)_{3}(\mathrm{~b})$, and $R \overline{3} m \mathrm{Bi}_{2}\left(\mathrm{Se}_{1-x} \mathrm{Te}_{x}\right)_{3}(\mathrm{c})$. The $P_{n}$ are sorted from highest to lowest, so that a straight line implies a homogenous probability distribution and substantial skew toward the upper left quadrant implies a preference for particular structures.

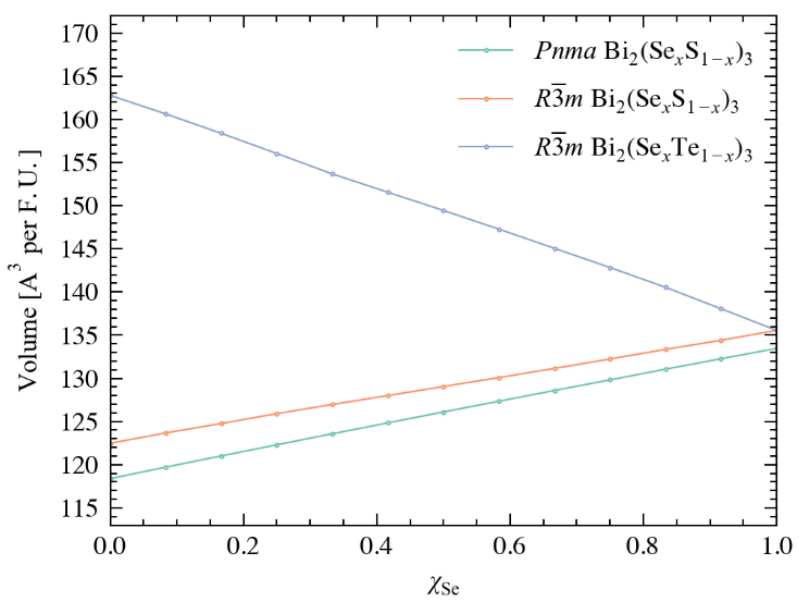

Figure 8 Predicted volume per formula unit of the Pnma $\mathrm{Bi}_{2}(\mathrm{~S}, \mathrm{Se})_{3}$ (green) and $R \overline{3} m \mathrm{Bi}_{2}(\mathrm{~S}, \mathrm{Se})_{3}$ (orange) and $\mathrm{Bi}_{2}(\mathrm{Se}, \mathrm{Te})_{3}$ (blue) alloys as a function of Se content $x_{\mathrm{Se}}$. 
Despite the skewed distribution of the $P_{n}$, the predicted cell volume as a function of composition $V(x)$ at $T_{\mathrm{F}}=800 \mathrm{~K}$ (Figure 8) is close to linear in the Se content, as would be expected from Vegard's law. This implies that there is a small variation in the volumes $V_{n}$ of individual structures.

Based on the mixing energies in Figure 6 we would expect $\mathrm{Bi}_{2}\left(\mathrm{Se}_{1-x} \mathrm{~S}_{x}\right)_{3}$ to form stable solid solutions in both the Pnma and $R \overline{3} m$ phases. Since we calculate the absolute free energies of each of the alloy compositions, we can also use our model to predict which of the two are most stable for a given composition and formation temperature (Figure 9). We find that at most temperatures and compositions the Pnma phase is the most energetically stable, with the $R \overline{3} m$ phase favored up to around $x=1 / 2(25-50 \% \mathrm{~S})$ below $\sim 550 \mathrm{~K}$, above which it is the most stable phase only very close to the SnSe endpoint $(x=0)$. Experimentally, $\mathrm{Bi}_{2}\left(\mathrm{Se}_{1-x} \mathrm{~S}_{x}\right)_{3}$ is reported to form a Pnma solid solution with compositions from $\mathrm{Bi}_{2} \mathrm{~S}_{3}(x=1)$ to the $\mathrm{Bi}_{40} \mathrm{Se}_{32} \mathrm{~S}_{28}(x=0.47),{ }^{17}$ Pnma phases with composition $\mathrm{Bi}_{2} \mathrm{Se}_{2} \mathrm{~S}$ and $\mathrm{Bi}_{2} \mathrm{SeS}_{2}(x=0.33 / 0.67),{ }^{19}$ and an $R \overline{3} m$ solid solution from $\mathrm{Bi}_{2} \mathrm{Se}_{3}$ to $\mathrm{Bi}_{40} \mathrm{Se}_{56} \mathrm{~S}_{4}(x=0.07) .{ }^{17}$ This is remarkably consistent with our predictions for synthesis temperatures above $\sim 600 \mathrm{~K}$.

Based on our results, alloying $\mathrm{Bi}_{2} \mathrm{Se}_{3}$ with a small amount of $\mathrm{Bi}_{2} \mathrm{~S}_{3}$ would constitute a reliable route to stabilizing the Pnma phase, whereas a low-temperature synthesis route may allow an $R \overline{3} m$ alloy with up to $\sim 50 \% \mathrm{~S}$ content to be formed. The former is notable as the Pnma phase is predicted to have a significantly lower thermal conductivity than the $R \overline{3} m$ phase, which would be beneficial for the thermoelectric properties. On the other hand, the latter is potentially also interesting as $R \overline{3} m \mathrm{Bi}_{2} \mathrm{Se}_{3}$ and $\mathrm{Bi}_{2} \mathrm{Te}_{3}$ are both topological insulators. ${ }^{17,72}$ 


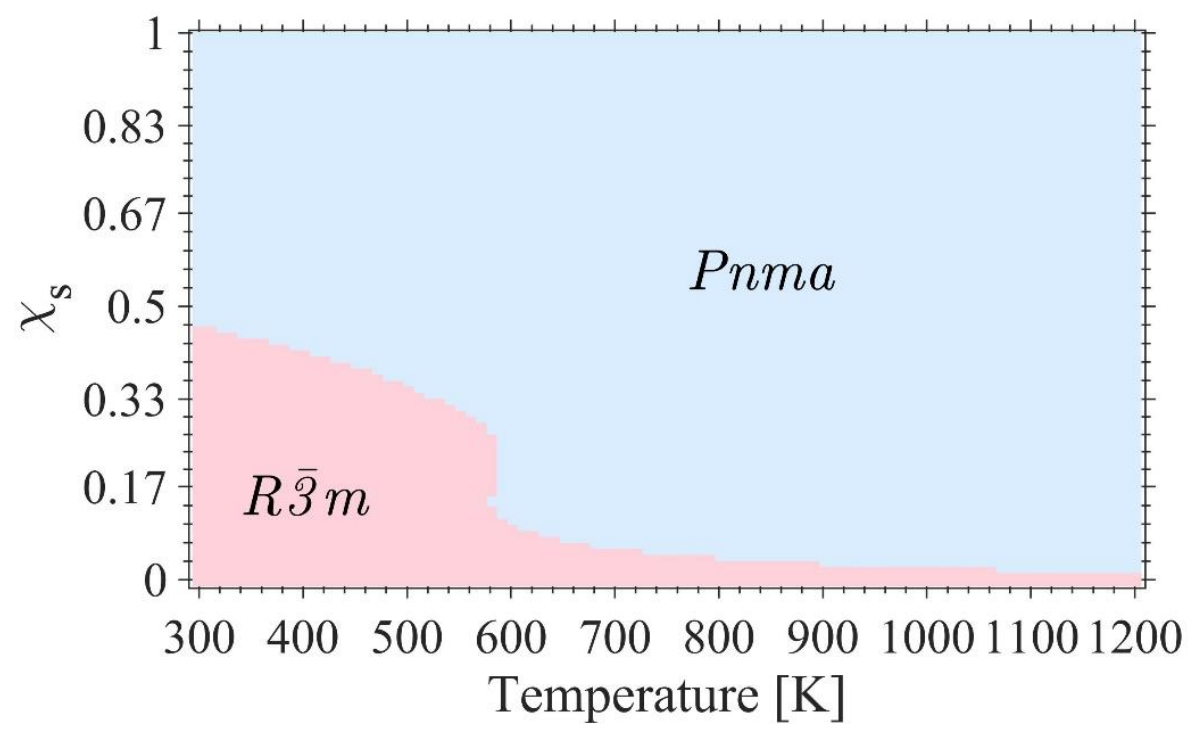

Figure 9 Predicted composition/temperature diagram for the $\mathrm{Bi}_{2}\left(\mathrm{Se}_{1-x} \mathrm{~S}_{x}\right)_{3}$ alloy showing the regions of the phase space where the Pnma and $R \overline{3} m$ phases have the lowest free energy.

We also note that while alloys with low S content may be unstable with respect to phase separation into Pnma $\mathrm{Bi}_{2} \mathrm{~S}_{3}$ and $R \overline{3} m \mathrm{Bi}_{2} \mathrm{Se}_{3}$ at lower temperature, the very different structures mean this would likely require significant atomic rearrangement, and alloys formed at high temperature and quenched may therefore remain kinetically stable.

Effect of Alloying on Thermal Transport. Finally, we investigate the thermal transport of the three alloy systems. In principle, one can calculate phonon spectra and thermal conductivities of individual structures in the alloy models and form a weighted average as per Eq. 12. However, given that large supercells are likely to be required for accurate results, this "brute force" approach is not feasible. ${ }^{45,46}$ 
The reduction in $\kappa_{\text {latt }}$ due to alloying and atomic substitution can be understood conceptually as arising from mass disorder and fluctuations in the strain field introduced by inhomogeneous chemical bonding. ${ }^{3}$ The former contribution can be modelled approximately using an isotope-scattering model, ${ }^{73}$ and we previously found that this approach provided a remarkably good prediction of the reduced $\kappa_{\text {latt }}$ in the Pnma $\operatorname{Sn}\left(\mathrm{S}_{0.1875} \mathrm{Se}_{0.8125}\right)$ alloy. ${ }^{46}$ A significant effect from strain-field fluctuations would be expected when the endpoints in the alloy show significantly different bond strengths. Since the bond strengths are captured by the force constants used to compute the harmonic phonon spectra and thermal conductivities, we can obtain some insight into this by recalculating the $\kappa_{\text {latt }}$ of the endpoint structures with the masses adjusted to the alloyed element. Taking Pnma $\mathrm{Bi}_{2}\left(\mathrm{Se}_{1-x} \mathrm{~S}_{x}\right)_{3}$ as an example, if setting the mass of $\mathrm{S}$ to $\mathrm{Se}$ in the $\mathrm{Bi}_{2} \mathrm{~S}_{3}$ structure gives a good reproduction of the $\kappa_{\text {latt }}$ of $\mathrm{Bi}_{2} \mathrm{Se}_{3}$, and vice versa, it would imply that the chemical bonding (force constants) in both phases are similar, and the primary difference in the $\kappa_{\text {latt }}$ is due to the different chalcogen masses. In this case, we might then expect an isotopescattering model to give a good reproduction of the thermal conductivity of the alloy.

Figures S12-S14 compare the averaged thermal conductivities $\kappa_{\text {ave }}$ of the endpoints of the three alloys to those obtained by adjusting the chalcogen mass to the other endpoint. Adjusting the mass of $\mathrm{S}$ in Pnma $\mathrm{Bi}_{2} \mathrm{~S}_{3}$ from $m_{\mathrm{S}}$ to $m_{\mathrm{Se}}$ overestimates the $\kappa_{\text {latt }}$ of $\mathrm{Bi}_{2} \mathrm{Se}_{3}$ by $42 \%$, while the reverse substitution in $\mathrm{Bi}_{2} \mathrm{Se}_{3}$ underestimates the $\kappa_{\text {latt }}$ of $\mathrm{Bi}_{2} \mathrm{~S}_{3}$ by $33 \%$. The equivalent procedure applied to the $R \overline{3} m$ phases produces closer agreement, with the $m_{\mathrm{S}} \rightarrow m_{\mathrm{Se}}$ adjustment in $\mathrm{Bi}_{2} \mathrm{~S}_{3}$ and the $m_{\mathrm{Se}} \rightarrow m_{\mathrm{S}}$ adjustment in $\mathrm{Bi}_{2} \mathrm{Se}_{3}$ leading to underestimates of 13 and $4 \%$ respectively. The $R \overline{3} m \mathrm{Bi}_{2} \mathrm{Se}_{3} / \mathrm{Bi}_{2} \mathrm{Te}_{3}$ pair appears to show the largest differences in bonding, with the $m_{\mathrm{Se}} \rightarrow m_{\mathrm{Te}}$ substitution in $\mathrm{Bi}_{2} \mathrm{Se}_{3}$ structure overestimating the $\kappa_{\text {latt }}$ of $\mathrm{Bi}_{2} \mathrm{Te}_{3}$ by a factor of two, and the reverse substitution in $\mathrm{Bi}_{2} \mathrm{Te}_{3}$ underestimating the $\kappa_{\text {latt }}$ of $\mathrm{Bi}_{2} \mathrm{Se}_{3}$ by $45 \%$. This analysis therefore implies 
that the impact of alloying on the $\kappa_{\text {latt }}$ of $R \overline{3} m \mathrm{Bi}_{2}\left(\mathrm{Se}_{1-x} \mathrm{~S}_{x}\right)_{3}$ may be driven by mass disorder, whereas strain-field fluctuations would likely be important in Pnma $\mathrm{Bi}_{2}\left(\mathrm{Se}_{1-x} \mathrm{~S}_{x}\right)_{3}$ and $R \overline{3} m \mathrm{Bi}_{2}\left(\mathrm{Se}_{1-}\right.$ $\left.{ }_{x} \mathrm{Te}_{x}\right)_{3}$.

To investigate further, we approximate the $\kappa_{\text {latt }}$ of the three alloys as a function of composition using the method employed in our previous work on the $\mathrm{Li}(\mathrm{No}, \mathrm{Mn}, \mathrm{Co}) \mathrm{O}_{2}(\mathrm{NMC})$ alloy system. ${ }^{74}$ We calculate the $\kappa_{\text {ave }}$ of both endpoint structures as the average mass $m_{\text {ave }}$ of the chalcogen is varied to the other endpoint:

$$
m_{\mathrm{ave}}=\sum_{i=1}^{n_{i}} a_{i} \times m_{i}
$$

where the sum runs over the $n_{i}$ species present at the disordered site with fractional occupation $a_{i}$ and mass $m_{i}$. We also use the isotope-scattering model in Ref. ${ }^{73}$ to model the effect of mass variance, which is parameterized by a mass variance $m_{\mathrm{var}}$ computed as:

$$
m_{\mathrm{var}}=\sum_{i=1}^{n_{i}} a_{i} \times\left(1-\frac{m_{i}}{m_{\mathrm{ave}}}\right)^{2}
$$

For each alloy, we obtain two predictions of $\kappa_{\text {ave }}$ as a function of composition (one for each endpoint), which we take as upper and lower bounds for predictions. 

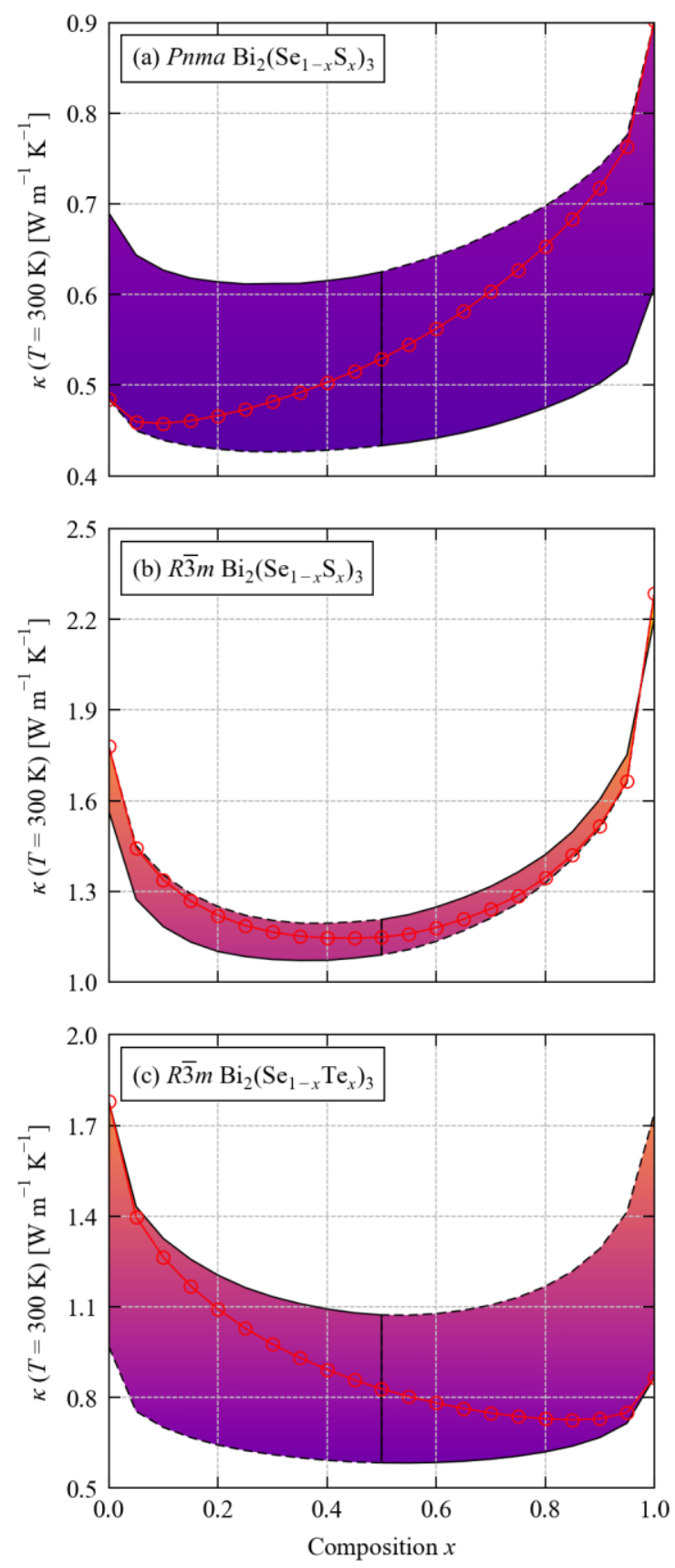

Figure 10 Averaged thermal conductivity $\kappa_{\mathrm{ave}}=\left(\kappa_{x x}+\kappa_{y y}+\kappa_{z z}\right) / 3$ at $T=300 \mathrm{~K}$ as a function of composition $x$ for Pnma $\mathrm{Bi}_{2}\left(\mathrm{Se}_{1-x} \mathrm{~S}_{x}\right)_{3}$ (a), $R \overline{3} m \mathrm{Bi}_{2}\left(\mathrm{Se}_{1-x} \mathrm{~S}_{x}\right)_{3}$ (b) and $R \overline{3} m \mathrm{Bi}_{2}\left(\mathrm{Se}_{1-x} \mathrm{Te}_{x}\right)_{3}$ (c) predicted using the force constants of the endpoint structures, an average mass, and an isotopescattering model to account for the effect of mass disorder. The shaded region on each plot shows 
the range of $\kappa_{\text {latt }}$ bounded by the two endpoints, the solid and dashed black outlines show the $\kappa_{\text {latt }}$ predicted by the closest and furthest endpoints, respectively, and the red lines with markers show interpolations between the two bounds.

The predictions of the $300 \mathrm{~K} \kappa_{\text {ave }}$ obtained from this model are shown in Figure 11. As expected, given the effects of the chalcogen-mass substitution on the $\kappa_{\text {latt }}$ of the endpoints, the predictions for Pnma $\mathrm{Bi}_{2}\left(\mathrm{Se}_{1-x} \mathrm{~S}_{x}\right)_{3}$ and $R \overline{3} m \mathrm{Bi}_{2}\left(\mathrm{Se}_{1-x} \mathrm{Te}_{x}\right)_{3}$ span wide ranges (Figure 11a/c), whereas those for $R \overline{3} m \mathrm{Bi}_{2}\left(\mathrm{Se}_{1-x} \mathrm{~S}_{x}\right)_{3}$ are much more sharply defined (Figure 11b). Interpolating between the boundaries, the minimum $\kappa_{\text {latt }}$ of the three alloys is predicted to occur at $\mathrm{Bi}_{2}\left(\mathrm{Se}_{0.9} \mathrm{~S}_{0.1}\right)$ $\left(0.46 \mathrm{~W} \mathrm{~m}^{-1} \mathrm{~K}^{-1}\right), \mathrm{Bi}_{2}\left(\mathrm{Se}_{0.55} \mathrm{~S}_{0.45}\right)_{3}\left(1.15 \mathrm{~W} \mathrm{~m}^{-1} \mathrm{~K}^{-1}\right)$, and $\mathrm{Bi}_{2}\left(\mathrm{Se}_{0.15} \mathrm{Te}_{0.85}\right)\left(0.73 \mathrm{~W} \mathrm{~m}^{-1} \mathrm{~K}^{-1}\right)$, which correspond to $5.5,36$ and $16 \%$ reductions relative to the $\mathrm{Bi}_{2} \mathrm{Se}_{3}$ and $\mathrm{Bi}_{2} \mathrm{Te}_{3}$ endpoints with the lower thermal conductivities. For $R \overline{3} m \mathrm{Bi}_{2}\left(\mathrm{Se}_{1-x} \mathrm{~S}_{x}\right)_{3}$, the magnitude of the reduction is very similar to the $40 \%$ reduction in the $\kappa_{\text {latt }}$ of $\mathrm{Sn}\left(\mathrm{S}_{0.1875} \mathrm{Se}_{0.8125}\right)$ relative to $\mathrm{SnSe}$ predicted by the massvariance model ${ }^{46}$ Although the predictions in the previous section indicate that $\operatorname{Bi}_{2}\left(\operatorname{Se}_{0.55} \mathrm{~S}_{0.45}\right)$ would adopt the Pnma structure, the steep decrease in $\kappa_{\text {latt }}$ with $\mathrm{S}$ content for $0<x<0.1$ suggests that alloying with a small amount of S may be beneficial to reducing the $\kappa_{\text {latt }}$ of $R \overline{3} m \mathrm{Bi}_{2} \mathrm{Se}_{3}$. Similarly, although $\mathrm{Bi}_{2}\left(\mathrm{Se}_{1-x} \mathrm{~S}_{x}\right)_{3}$ with $x=0.1$ may preferentially adopt the $R \overline{3} m$ structure, compositions up to around $x=0.3$ are predicted to yield a similar or lower $\kappa_{\text {latt }}$ than Pnma $\mathrm{Bi}_{2} \mathrm{Se}_{3}$, which is itself significantly lower than the thermal conductivity of the $R \overline{3} m$ phase.

Despite the approximations in this model, several of the predictions are nonetheless a reasonable qualitative match to the experimental measurements in Ref. ${ }^{19}$. The measurements on the $\mathrm{Bi}_{2}(\mathrm{~S}, \mathrm{Se})_{3}$ system indicate that $\mathrm{Bi}_{2} \mathrm{~S}_{2} \mathrm{Se}$ and $\mathrm{Bi}_{2} \mathrm{SSe}_{2}$, which both adopt the Pnma phase, have 
a lower $\kappa_{\text {latt }}$ than $\mathrm{Bi}_{2} \mathrm{~S}_{3}$. Similarly, the measurements on $\mathrm{Bi}_{2}(\mathrm{Se}, \mathrm{Te})_{3}$ predict a minimum in the $\kappa_{\text {latt }}$ for $\mathrm{Bi}_{2} \mathrm{Se}_{0.3} \mathrm{Te}_{2.7}$, a reduced $\kappa_{\text {latt }}$ for $\mathrm{Bi}_{2} \mathrm{SeTe}_{2}$, and an increased $\kappa_{\text {latt }}$ for $\mathrm{Bi}_{2} \mathrm{Se}_{2} \mathrm{Te}$ compared to $\mathrm{Bi}_{2} \mathrm{Te}_{3}$. The interpolation predicts the minimum to occur at $\mathrm{Bi}_{2} \mathrm{Se}_{0.15} \mathrm{Te}_{0.85}$, which is between the two compositions measured to have $\kappa_{\text {latt }}$ lower than $\mathrm{Bi}_{2} \mathrm{Te}_{3}$, and the model also predicts an increase in $\kappa_{\text {latt }}$ above the $\mathrm{Bi}_{2} \mathrm{Te}_{3}$ endpoint at $\mathrm{Bi}_{2} \mathrm{Se}_{2} \mathrm{Te}(x=1 / 3)$. We therefore conclude that this model can make useful qualitative, if not quantitative, predictions.

As noted above, with a $T_{\mathrm{F}}$ of $800 \mathrm{~K}$ we find that the $\mathrm{Bi}_{2} \mathrm{SeTe}_{2}$ composition is heavily skewed towards a small number of structures, which makes it feasible to compute an approximate $\kappa_{\text {latt }}$ from explicit calculations on these structures. We select the two most probable structures, with $P_{n}=0.42$ and 0.2 , and compute an averaged $\kappa_{\text {latt }}$ as a function of temperature from a partial weighted sum according to (c.f. Eq. 12):

$$
\bar{\kappa}_{\mathrm{ave}}\left(T_{\mathrm{F}} ; T\right)=\frac{\sum_{n=1}^{n_{x}^{\prime}} P_{n}\left(T_{\mathrm{F}}\right) \times \kappa_{\mathrm{ave}, n}(T)}{\sum_{n=1}^{n_{x}^{\prime}} P_{n}\left(T_{\mathrm{F}}\right)}
$$

where the sum runs over a subset $n_{x}^{\prime}=2$ of the $n_{x}=21$ structures in the model and we have omitted the dependence on the composition $x$ for brevity. Figure 11 shows the predicted temperature dependence of the $\kappa_{\text {latt }}$ of $\mathrm{Bi}_{2} \mathrm{SeTe}_{3}$ obtained for $T_{\mathrm{F}}=800 \mathrm{~K}$. We also compare this to two models using the $\mathrm{Bi}_{2} \mathrm{Te}_{3}$ force constants, viz. one where we change the mass of the Te atom at the interior $\mathrm{Chal}^{(1)}$ site from $m_{\mathrm{Te}}$ to $m_{\mathrm{Se}}$, and a second where we use an average mass and isotope-scattering model to approximate a random distribution of Se and Te over both chalcogen positions. The latter third model is equivalent to the analysis in Figure 10. 


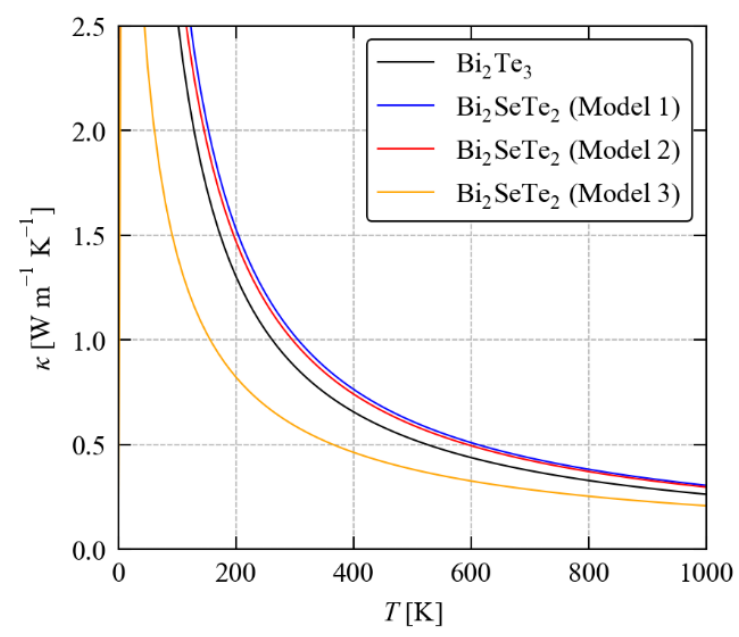

Figure 11 Predicted average thermal conductivity $\kappa_{\mathrm{ave}}=\left(\kappa_{x x}+\kappa_{y y}+\kappa_{z z}\right) / 3$ as a function of temperature for $\mathrm{Bi}_{2} \mathrm{Te}_{3}$ (black) and $\mathrm{Bi}_{2} \mathrm{Se}_{2} \mathrm{Te}_{2}$ estimated with three different models. Model 1 (blue): Partial weighted average over the two most probable structures in the alloy model. Model 2 (red): $\mathrm{Bi}_{2} \mathrm{Te}_{3}$ with the mass of the interior $\mathrm{Chal}^{(1)}$ site changed from $m_{\mathrm{Te}}$ to $m_{\mathrm{Se}}$. Model 3 (orange): $\mathrm{Bi}_{2} \mathrm{Te}_{3}$ with an isotope-scattering model used to model a random distribution of $\mathrm{Se}$ and Te over both chalcogen positions.

Surprisingly, and in contrast to experiments, we find that the partial weighted sum predicts a $\kappa_{\text {latt }}$ higher than $\mathrm{Bi}_{2} \mathrm{Te}_{3}$. The most probable of the two structures with $P_{n}=0.42$ corresponds to an ordered phase where the $\mathrm{Chal}^{(1)}$ and $\mathrm{Chal}^{(2)}$ sites are fully occupied by Se and Te atoms. The calculated in-plane and out-of-plane $\kappa_{\text {latt }}$ of this configuration at $T=300 \mathrm{~K}$ is 2.55 and $1.37 \mathrm{~W} \mathrm{~m}^{-}$ ${ }^{1} \mathrm{~K}^{-1}$, compared to $1.01 / 0.61$ and $2.26 / 0.94 \mathrm{~W} \mathrm{~m}^{-1} \mathrm{~K}^{-1}$ in $\mathrm{Bi}_{2} \mathrm{Te}_{3}$ and $\mathrm{Bi}_{2} \mathrm{Se}_{3}$ respectively. As discussed previously, Se would likely show stronger ionic bonding in the $\mathrm{Chal}^{(1)}$ site and Te would 
show stronger vdW interactions in the $\mathrm{Chal}^{(2)}$ position, so this structural arrangement of $\mathrm{Bi}_{2} \mathrm{SeTe}_{2}$ may in fact optimize the chemical bonding strength compared to either endpoint.

The theoretical study on monolayer $\mathrm{Bi}_{2} \mathrm{SeTe}_{2}$ with the same structural ordering in Ref. ${ }^{48}$ reports a $\kappa_{\text {latt }}$ of $1.96 \mathrm{~W} \mathrm{~m}^{-1} \mathrm{~K}^{-1}$, which is $23 \%$ lower than our calculated in-plane value. The similar theoretical studies in Ref. ${ }^{42}$ report room-temperature thermal conductivities of 3.92 and $1.35 \mathrm{~W} \mathrm{~m}^{-1} \mathrm{~K}^{-1}$, depending on whether or not spin-orbit coupling (SOC) is included in the calculations, and our value falls between these two extremes. (We note in passing, however, that the large effect of SOC suggested by the latter study is somewhat surprising, especially given the small effect on the phonon dispersion.)

The second most probable structure $\left(P_{n}=0.2\right)$, on the other hand, has a much smaller $\kappa_{\text {ave }}$ of $0.62 \mathrm{~W} \mathrm{~m}^{-1} \mathrm{~K}^{-1}$, which is lower than $\mathrm{Bi}_{2} \mathrm{Te}_{3}$. Since the other 19 of the 21 structures in the alloy model would also show some level of chalcogen disorder compared to the ideal arrangement, we would expect these to have a $\kappa_{\text {latt }}$ closer to the second structure, which would in turn bring the complete weighted sum closer to the $\sim 10 \%$ smaller $\kappa_{\text {latt }}$ relative to $\mathrm{Bi}_{2} \mathrm{Te}_{3}$ measured in Ref. ${ }^{19}$.

The second model for the $\kappa_{\text {latt }}$ of $\mathrm{Bi}_{2} \mathrm{SeTe}_{2}$, using the $\mathrm{Bi}_{2} \mathrm{Te}_{3}$ structure and force constants but with the mass of the Chal ${ }^{(1)}$ ion adjusted from $m_{\mathrm{Te}}$ to $m_{\mathrm{Se}}$, gives remarkably similar results to the partial weighted sum. This model incorporates the increased $\mathrm{Chal}^{(1)}$ mass but neglects the enhanced bond strength, and thus gives a result intermediate between the two explicit calculations in the partial weighted sum. Finally, the third model, assuming a random distribution of Se and Te over the three chalcogen sites, predicts a $300 \mathrm{~K}_{\text {ave }}$ of $0.59 \mathrm{~W} \mathrm{~m}^{-1} \mathrm{~K}^{-1}$, which is a $\sim 30 \%$ reduction compared to $\mathrm{Bi}_{2} \mathrm{Te}_{3}$. This larger than the $10 \%$ observed experimentally, and emphasizes the impact of the inhomogeneous distribution of chalcogen atoms predicted in the previous section. 


\section{CONCLUSIONS}

In summary, we have performed a detailed study of the structural dynamics and thermal transport in the bismuth chalcogenides $\mathrm{Bi}_{2} \mathrm{~S}_{3}, \mathrm{Bi}_{2} \mathrm{Se}_{3}$ and $\mathrm{Bi}_{2} \mathrm{Te}_{3}$ and their alloys.

The orthorhombic Pnma and rhombohedral $R \overline{\mathbf{3}} m$ phases of the three chalcogenides are dynamically stable, whereas the tetragonal $P 4_{2} / n m c$ phase of $\mathrm{Bi}_{2} \mathrm{Se}_{3}$ and the alternative rhombohedral $R \overline{\mathbf{3}} m^{*}$ phase of $\mathrm{Bi}_{2} \mathrm{Te}_{3}$, both of which are reported under non-ambient conditions, display phonon instabilities. Due to the differing interactions between the Bi 6 s lone pair and the anion valence $\mathrm{p}$ orbitals, the Pnma phase of $\mathrm{Bi}_{2} \mathrm{~S}_{3}$ is energetically preferred, whereas the heavier chalcogenides prefer the $R \overline{\mathbf{3}} m$ phase. The Pnma phase is predicted to be metastable for $\mathrm{Bi}_{2} \mathrm{Se}_{3}$, with vibrational contributions to the Helmholtz free energy bringing it closer to the convex hull at elevated temperature.

Thermal conductivities calculated using the relaxation-time approximation show good agreement with experimental measurements and highlight the lower thermal conductivity associated with the Pnma structure. Analysis of the modal contributions to the $\boldsymbol{\kappa}_{\text {latt }}$ attributes this to be due to a combination of reduced group velocities, indicative of weaker chemical bonding, and shorter mode lifetimes, indicative of enhanced anharmonicity, in the orthorhombic phase.

A thermodynamic model for the Pnma $\mathrm{Bi}_{2}\left(\mathrm{Se}_{1-x} \mathrm{~S}_{x}\right)_{3}$ alloy predicts near-ideal mixing behavior, with the formation of a structurally-homogeneous solid solution favored by the configurational entropy. On the other hand, an energetic preference for smaller and larger chalcogens to occupy the interior and exterior positions in the quintuple layers of the $R \overline{3} m$ structure results in notable deviation from ideality, particularly for $\mathrm{Bi}_{2}\left(\mathrm{Se}_{1-x} \mathrm{Te}_{x}\right)_{3}$. A prediction of the 
conditions under which the competing Pnma and $R \overline{3} m$ phases of $\mathrm{Bi}_{2}\left(\mathrm{Se}_{1-x} \mathrm{~S}_{x}\right)_{3}$ are expected to form shows remarkable agreement with experiment, and suggests that the stability window over which the $R \overline{3} m$ alloy forms could possibly be extended using low-temperature synthesis routes.

Finally, analysis of the $\kappa_{\text {latt }}$ of the alloys using approximate models suggests that the reduction in the thermal conductivity of $R \overline{3} m \mathrm{Bi}_{2}\left(\mathrm{Se}_{1-x} \mathrm{~S}_{x}\right)_{3}$ is likely to be driven predominantly by mass disorder, whereas strain-field fluctuations due to inhomogeneous chemical bonding play a significant role in the reduced $\kappa_{\text {latt }}$ of Pnma $\mathrm{Bi}_{2}\left(\mathrm{Se}_{1-x} \mathrm{~S}_{x}\right)_{3}$ and $R \overline{3} m \mathrm{Bi}_{2}\left(\mathrm{Se}_{1-x} \mathrm{Te}_{x}\right)_{3}$. Nonetheless, we find that using an isotope-scattering model to predict the effect of mass disorder allows for useful qualitative predictions. A more detailed analysis of the thermal transport in $\mathrm{Bi}_{2} \mathrm{SeTe}_{2}$ indicates that disordered occupation of the chalcogen atoms away from the preferred sites, favored by configurational entropy, is likely to be an important factor in the reduced $\kappa_{\text {latt }}$ of this compound compared to $\mathrm{Bi}_{2} \mathrm{Te}_{3}$ observed in experiments.

Overall, this study highlights the utility of first-principles modelling in predicting and understanding the stability, structural dynamics and heat transport in thermoelectric materials, for predicting the structure and energetics of moderately-complex alloys, and for establishing the impact of alloying on the thermal conductivity. Given that alloying is an accepted strategy for optimizing the performance of thermoelectric materials, we hope that this work and similar studies will provide useful guidance to ongoing work on the bismuth chalcogenides and related systems.

\section{ASSOCIATED CONTENT}

Supporting Information. Supporting information is available online at [URL to be added by publisher]: calculated phonon dispersion and PDoS curves for Pnma and $R \overline{3} m \mathrm{Bi}_{2} \mathrm{~S}_{3}, P n m a, R \overline{3} m$ 
and $P 4_{2} / n m c \mathrm{Bi}_{2} \mathrm{Se}_{3}$, and $P n m a, R \overline{3} m$ and $R \overline{3} m^{*} \mathrm{Bi}_{2} \mathrm{Te}_{3}$; breakdown of the mixing energies of Pnma $\mathrm{Bi}_{2}\left(\mathrm{Se}_{1-x} \mathrm{~S}_{x}\right)_{3}$, and $R \overline{3} m \mathrm{Bi}_{2}\left(\mathrm{Se}_{1-x} \mathrm{~S}_{x}\right)_{3}$ and $\mathrm{Bi}_{2}\left(\mathrm{Se}_{1-x} \mathrm{Te}_{x}\right)_{3}$ into contributions from internal energy and entropy; and comparison of the thermal conductivity of the Pnma $\mathrm{Bi}_{2} \mathrm{~S}_{3} / \mathrm{Bi}_{2} \mathrm{Se}_{3}, R \overline{3} m$ $\mathrm{Bi}_{2} \mathrm{~S}_{3} / \mathrm{Bi}_{2} \mathrm{Se}_{3}$ and $R \overline{3} m \mathrm{Bi}_{2} \mathrm{Se}_{3} / \mathrm{Bi}_{2} \mathrm{Te}_{3}$ endpoint pairs with chalcogen mass substitution.

Data-Access Statement. Raw data from this study including optimized structures, input and output files from the lattice-dynamics and thermal-conductivity calculations, and data from the alloy models, is available to download free of charge from an online repository at [URL to be added on acceptance].

\section{AUTHOR INFORMATION}

\section{Corresponding Author}

* E-Mail: jonathan.skelton@manchester.ac.uk

\section{Author Contributions}

The manuscript was written through contributions of all authors. All authors have given approval to the final version of the manuscript.

\section{Conflict of Interest}

The authors declare no conflicts of interest.

\section{ACKNOWLEDGMENT}

IP is supported by a UoM PhD studentship. JMS is supported by a UK Research and Innovation (UKRI) Future Leaders Fellowship (MR/T043121/1), and previously held a University of Manchester Presidential Fellowship. The majority of the calculations were performed on the UK Archer HPC facility, via the UK Materials Chemistry Consortium, which is funded by the UK 
Engineering and Physical Sciences Research Council (EP/L000202, EP/R029431). Some calculations were also performed using the UoM Computational Shared Facility, which is maintained by the UoM IT services.

\section{ABBREVIATIONS}

DFT - density-functional theory; RTA - (single-mode) relaxation-time approximation; CRTA constant relaxation-time approximation.

\section{REFERENCES}

(1) Stern, P. C.; Sovacool, B. K.; Dietz, T. Towards a Science of Climate and Energy Choices. Nat. Clim. Chang. 201666 2016, 6(6), 547-555. https://doi.org/10.1038/NCLIMATE3027.

(2) Freer, R.; Powell, A. V. Realising the Potential of Thermoelectric Technology: A Roadmap. Journal of Materials Chemistry C. Royal Society of Chemistry January 2, 2020, pp 441463. https://doi.org/10.1039/c9tc05710b.

(3) Tan, G.; Zhao, L.-D.; Kanatzidis, M. G. Rationally Designing High-Performance Bulk Thermoelectric Materials. Chem. Rev. 2016, 116 (19), 12123-12149. https://doi.org/10.1021/acs.chemrev.6b00255.

(4) Sootsman, J. R.; Chung, D. Y.; Kanatzidis, M. G. New and Old Concepts in Thermoelectric Materials. Angew. Chemie Int. Ed. 2009, 48 (46), 8616-8639. https://doi.org/10.1002/anie.200900598.

(5) Zeier, W. G.; Zevalkink, A.; Gibbs, Z. M.; Hautier, G.; Kanatzidis, M. G.; Snyder, G. J. Thinking Like a Chemist: Intuition in Thermoelectric Materials. Angew. Chemie Int. Ed. 2016, 55 (24), 6826-6841. https://doi.org/10.1002/ANIE.201508381. 
(6) Biswas, K.; He, J.; Blum, I. D.; Wu, C.-I.; Hogan, T. P.; Seidman, D. N.; Dravid, V. P.; Kanatzidis, M. G. High-Performance Bulk Thermoelectrics with All-Scale Hierarchical Architectures. Nature 2012, 489 (7416), 414-418. https://doi.org/10.1038/nature11439.

(7) Delaire, O.; Ma, J.; Marty, K.; May, A. F.; McGuire, M. A.; Du, M.-H.; Singh, D. J.; Podlesnyak, A.; Ehlers, G.; Lumsden, M. D.; et al. Giant Anharmonic Phonon Scattering in PbTe. Nat. Mater. 2011, 10 (8), 614-619. https://doi.org/10.1038/nmat3035.

(8) Pei, Y.; Shi, X.; LaLonde, A.; Wang, H.; Chen, L.; Snyder, G. J. Convergence of Electronic Bands for High Performance Bulk Thermoelectrics. Nat. 20114737345 2011, 473 (7345), 66-69. https://doi.org/10.1038/NATURE09996.

(9) Gibbs, Z. M.; Kim, H.; Wang, H.; White, R. L.; Drymiotis, F.; Kaviany, M.; Snyder, G. J. Temperature Dependent Band Gap in $\mathrm{PbX}(\mathrm{X}=\mathrm{S}, \mathrm{Se}, \mathrm{Te})$. Appl. Phys. Lett. 2013, 103 (26), 262109. https://doi.org/10.1063/1.4858195.

(10) Zhao, L.-D.; Lo, S.-H.; Zhang, Y.; Sun, H.; Tan, G.; Uher, C.; Wolverton, C.; Dravid, V. P.; Kanatzidis, M. G. Ultralow Thermal Conductivity and High Thermoelectric Figure of Merit in SnSe Crystals. Nature 2014, 508, 373-377. https://doi.org/10.1038/nature13184.

(11) Li, C. W.; Hong, J.; May, A. F.; Bansal, D.; Chi, S.; Hong, T.; Ehlers, G.; Delaire, O. Orbitally Driven Giant Phonon Anharmonicity in SnSe. Nat. Phys. 2015, 11, 1063-1069. https://doi.org/10.1038/nphys3492.

(12) Skelton, J. M.; Burton, L. A.; Parker, S. C.; Walsh, A.; Kim, C.-E.; Soon, A.; Buckeridge, J.; Sokol, A. A.; Catlow, C. R. A.; Togo, A.; et al. Anharmonicity in the High-Temperature Cmcm Phase of SnSe: Soft Modes and Three-Phonon Interactions. Phys. Rev. Lett. 2016, 
117 (7), 075502. https://doi.org/10.1103/PhysRevLett.117.075502.

(13) Zhao, L.-D.; Tan, G.; Hao, S.; He, J.; Pei, Y.; Chi, H.; Wang, H.; Gong, S.; Xu, H.; Dravid, V. P.; et al. Ultrahigh Power Factor and Thermoelectric Performance in Hole-Doped SingleCrystal SnSe. Science 2016, 351 (6269), 141-144. https://doi.org/10.1126/science.aad3749.

(14) Aseginolaza, U.; Bianco, R.; Monacelli, L.; Paulatto, L.; Calandra, M.; Mauri, F.; Bergara, A.; Errea, I. Phonon Collapse and Second-Order Phase Transition in Thermoelectric SnSe. Phys. Rev. Lett. 2019, 122 (7), 075901. https://doi.org/10.1103/PhysRevLett.122.075901.

(15) Witting, I. T.; Chasapis, T. C.; Ricci, F.; Peters, M.; Heinz, N. A.; Hautier, G.; Snyder, G. J. The Thermoelectric Properties of Bismuth Telluride. Advanced Electronic Materials. Blackwell Publishing Ltd June 1, 2019, $\quad$ p 1800904. https://doi.org/10.1002/aelm.201800904.

(16) Lee, S.; Esfarjani, K.; Luo, T.; Zhou, J.; Tian, Z.; Chen, G. Resonant Bonding Leads to Low Lattice Thermal Conductivity. Nat. Commun. $2014 \quad 51 \quad$ 2014, 5 (1), $1-8$. https://doi.org/10.1038/NCOMMS4525.

(17) Heremans, J. P.; Cava, R. J.; Samarth, N. Tetradymites as Thermoelectrics and Topological Insulators. Nat. Rev. Mater. $2017 \quad 210 \quad \mathbf{2 0 1 7}, \quad 2 \quad$ (10), $1-21$. https://doi.org/10.1038/NATREVMATS.2017.49.

(18) Goldsmid, H. J. Recent Studies of Bismuth Telluride and Its Alloys. J. Appl. Phys. 1961, 32 (10), 2198. https://doi.org/10.1063/1.1777042.

(19) Liu, W.; Lukas, K. C.; McEnaney, K.; Lee, S.; Zhang, Q.; Opeil, C. P.; Chen, G.; Ren, Z. 
Studies on the Bi2Te3-Bi2Se3-Bi2S3 System for Mid-Temperature Thermoelectric Energy Conversion. Energy Environ. Sci. 2013, $6 \quad$ (2), 552-560. https://doi.org/10.1039/C2EE23549H.

(20) Tan, M.; Deng, Y.; Hao, Y. Enhancement of Thermoelectric Properties Induced by Oriented Nanolayer in Bi2Te2.7Se0.3 Columnar Films. Mater. Chem. Phys. 2014, 146 (1-2), 153158. https://doi.org/10.1016/J.MATCHEMPHYS.2014.03.014.

(21) Tan, M.; Hao, Y.; Wang, G. Improvement of Thermoelectric Properties Induced by Uniquely Ordered Lattice Field in Bi2Se0.5Te2.5 Pillar Array. J. Solid State Chem. 2014, 215, 219-224. https://doi.org/10.1016/J.JSSC.2014.04.005.

(22) Liu, R.; Tan, X.; Ren, G.; Liu, Y.; Zhou, Z.; Liu, C.; Lin, Y.; Nan, C. Enhanced Thermoelectric Performance of Te-Doped Bi2Se3-xTex Bulks by Self-Propagating HighTemperature Synthesis. Cryst. 2017, Vol. 7, Page 257 2017, 7 (9), 257. https://doi.org/10.3390/CRYST7090257.

(23) Soni, A.; Yanyuan, Z.; Ligen, Y.; Aik, M. K. K.; Dresselhaus, M. S.; Xiong, Q. Enhanced Thermoelectric Properties of Solution Grown Bi2Te3-XSex Nanoplatelet Composites. Nano Lett. 2012, 12 (3), 1203-1209. https://doi.org/10.1021/NL2034859.

(24) Prokofieva, L. V.; Pshenay-Severin, D. A.; Konstantinov, P. P.; Shabaldin, A. A. Optimum Composition of a Bi 2 Te 3 - x Se x Alloy for the $n$-Type Leg of a Thermoelectric $\begin{array}{llllll}\text { Generator. } \quad \text { Semicond. } & 2009 & 438 & \mathbf{2 0 0 9}, & 43 & \text { (8), }\end{array}$ https://doi.org/10.1134/S1063782609080016.

(25) Madsen, G. K. H.; Singh, D. J. BoltzTraP. A Code for Calculating Band-Structure 
Dependent Quantities. Comput. Phys. Commun. 2006, 175 (1), 67-71. https://doi.org/10.1016/j.cpc.2006.03.007.

(26) Ganose, A. M.; Park, J.; Faghaninia, A.; Woods-Robinson, R.; Persson, K. A.; Jain, A. Efficient Calculation of Carrier Scattering Rates from First Principles. Nat. Commun. 2021 121 2021, 12 (1), 1-9. https://doi.org/10.1038/S41467-021-22440-5.

(27) Togo, A.; Tanaka, I. First Principles Phonon Calculations in Materials Science. Scr. Mater. 2015, 108, 1-5.

(28) Togo, A.; Chaput, L.; Tanaka, I. Distributions of Phonon Lifetimes in Brillouin Zones. Phys. Rev. B 2015, 91 (9), 094306. https://doi.org/10.1103/PhysRevB.91.094306.

(29) Li, W.; Carrete, J.; Katcho, N. A.; Mingo, N. ShengBTE: A Solver of the Boltzmann Transport Equation for Phonons. Comput. Phys. Commun. 2014, 185 (6), 1747-1758. https://doi.org/10.1016/j.cpc.2014.02.015.

(30) Carrete, J.; Vermeersch, B.; Katre, A.; van Roekeghem, A.; Wang, T.; Madsen, G. K. H.; Mingo, N. AlmaBTE: A Solver of the Space-Time Dependent Boltzmann Transport Equation for Phonons in Structured Materials. Comput. Phys. Commun. 2017, 220, 351362. https://doi.org/10.1016/j.cpc.2017.06.023.

(31) Whalley, L. D.; Skelton, J. M.; Frost, J. M.; Walsh, A. Phonon Anharmonicity, Lifetimes, and Thermal Transport in CH3NH3PbI3 from Many-Body Perturbation Theory. Phys. Rev. B 2016, 94 (22). https://doi.org/10.1103/PhysRevB.94.220301.

(32) Gold-Parker, A.; Gehring, P. M.; Skelton, J. M.; Smith, I. C.; Parshall, D.; Frost, J. M.; 
Karunadasa, H. I.; Walsh, A.; Toney, M. F. Acoustic Phonon Lifetimes Limit Thermal Transport in Methylammonium Lead Iodide. Proc. Natl. Acad. Sci. U. S. A. 2018, 115 (47), 11905-11910. https://doi.org/10.1073/pnas.1812227115.

(33) Aseginolaza, U.; Bianco, R.; Monacelli, L.; Paulatto, L.; Calandra, M.; Mauri, F.; Bergara, A.; Errea, I. Strong Anharmonicity and High Thermoelectric Efficiency in HighTemperature SnS from First Principles. Phys. Rev. B 2019, 100 (21), 214307. https://doi.org/10.1103/PhysRevB.100.214307.

(34) Rahim, W.; Skelton, J. M.; Scanlon, D. O. $\alpha-\mathrm{Bi}_{2} \mathrm{Sn}_{2} \mathrm{O}_{7}$ : A Potential Room Temperature nType Oxide Thermoelectric. J. Mater. Chem. A 2020, 8 (32), 16405-16420. https://doi.org/10.1039/d0ta03945d.

(35) Mishra, S. K.; Satpathy, S.; Jepsen, O. Electronic Structure and Thermoelectric Properties of Bismuth Telluride and Bismuth Selenide. J. Phys. Condens. Matter 1997, 9 (2), 461. https://doi.org/10.1088/0953-8984/9/2/014.

(36) Huang, B.-L.; Kaviany, M. Ab Initio and Molecular Dynamics Predictions for Electron and Phonon Transport in Bismuth Telluride. Phys. Rev. B 2008, 77 (12), 125209. https://doi.org/10.1103/PhysRevB.77.125209.

(37) Park, M. S.; Song, J.-H.; Medvedeva, J. E.; Kim, M.; Kim, I. G.; Freeman, A. J. Electronic Structure and Volume Effect on Thermoelectric Transport in <span Class. Phys. Rev. B 2010, 81 (15), 155211. https://doi.org/10.1103/PhysRevB.81.155211.

(38) Luo, X.; Sullivan, M. B.; Quek, S. Y. First-Principles Investigations of the Atomic, Electronic, and Thermoelectric Properties of Equilibrium and Strained Bi<span Class. Phys. 
Rev. B 2012, 86 (18), 184111. https://doi.org/10.1103/PhysRevB.86.184111.

(39) Cheng, L.; Liu, H. J.; Zhang, J.; Wei, J.; Liang, J. H.; Shi, J.; Tang, X. F. Effects of van Der Waals Interactions and Quasiparticle Corrections on the Electronic and Transport Properties of <span Class. Phys. Rev. B 2014, $90 \quad$ (8), 085118. https://doi.org/10.1103/PhysRevB.90.085118.

(40) Sharma, S.; Schwingenschlögl, U. Thermoelectric Response in Single Quintuple Layer Bi2Te3. ACS Energy Lett. 2016, 1 (4), 875-879. https://doi.org/10.1021/ACSENERGYLETT.6B00289.

(41) Wang, B.; Niu, X.; Ouyang, Y.; Zhou, Q.; Wang, J. Ultrathin Semiconducting Bi2Te2S and Bi2Te2Se with High Electron Mobilities. J. Phys. Chem. Lett. 2018, 9 (3), 487-490. https://doi.org/10.1021/ACS.JPCLETT.7B03036.

(42) Rashid, Z.; Nissimagoudar, A. S.; Li, W. Phonon Transport and Thermoelectric Properties of Semiconducting Bi2Te2X (X = S, Se, Te) Monolayers. Phys. Chem. Chem. Phys. 2019, 21 (10), 5679-5688. https://doi.org/10.1039/C8CP05793A.

(43) Xia, Q.; Xu, Q.; Xu, B.; Yi, L. Ab Initio Prediction of Thermoelectric Performance of Monolayer BiSbTe3. J. Solid State Chem. 2021, 296, 121963. https://doi.org/10.1016/J.JSSC.2021.121963.

(44) Gunn, D. S. D.; Skelton, J. M.; Burton, L. A.; Metz, S.; Parker, S. C. Thermodynamics, Electronic Structure, and Vibrational Properties of $\mathrm{Sn}_{n}\left(\mathrm{~S}_{1-x} \mathrm{Se}_{x}\right)_{m}$ Solid Solutions for Energy Applications. Chem. Mater. 2019, $31 \quad$ (10), 3672-3685. https://doi.org/10.1021/acs.chemmater.9b00362. 
(45) Skelton, J. M. Lattice Dynamics of Pnma $\mathrm{Sn}\left(\mathrm{S}_{1-x} \mathrm{Se}_{x}\right)$ Solid Solutions: Energetics, Phonon Spectra and Thermal Transport. J. Phys. Energy 2020, 2 (2), 025006. https://doi.org/10.1088/2515-7655/AB7839.

(46) Skelton, J. M. Approximate Models for the Lattice Thermal Conductivity of Alloy Thermoelectrics. J. Mater. Chem. C 2021. https://doi.org/10.1039/d1tc02026a.

(47) Ektarawong, A.; Alling, B. Stability of $\mathrm{SnSe}_{1-x} \mathrm{~S}_{x}$ Solid Solutions Revealed by FirstPrinciples Cluster Expansion. J. Phys. Condens. Matter 2018, 30 (29), 29 LT01. https://doi.org/10.1088/1361-648X/aacb9c.

(48) Xu, B.; Song, L.; Peng, G.; Zhang, J.; Ma, S.; Wang, Y.; Wang, Y. Thermoelectric Performance of Monolayer Bi2Te2Se of Ultra Low Lattice Thermal Conductivity. Phys. Lett. A 2019, 383 (28), 125864. https://doi.org/10.1016/J.PHYSLETA.2019.125864.

(49) Kresse, G.; Furthmüller, J. Efficient Iterative Schemes for Ab Initio Total-Energy Calculations Using a Plane-Wave Basis Set. Phys. Rev. B 1996, 54 (16), 11169. https://doi.org/10.1103/PhysRevB.54.11169.

(50) Kresse, G.; Joubert, D. From Ultrasoft Pseudopotentials to the Projector Augmented-Wave Method. Phys. Rev. B 1999, 59(3), 1758-1775. https://doi.org/10.1103/PhysRevB.59.1758.

(51) Jain, A.; Ong, S. P.; Hautier, G.; Chen, W.; Richards, W. D.; Dacek, S.; Cholia, S.; Gunter, D.; Skinner, D.; Ceder, G.; et al. Commentary: The Materials Project: A Materials Genome Approach to Accelerating Materials Innovation. APL Mater. 2013, 1, 011002. https://doi.org/10.1063/1.4812323. 
(52) Monkhorst, H. J.; Pack, J. D. Special Points for Brillouin-Zone Integrations. Phys. Rev. B 1976, 13 (12), 5188-5192. https://doi.org/10.1103/PhysRevB.13.5188.

(53) Perdew, J. P.; Ruzsinszky, A.; Csonka, G. I.; Vydrov, O. A.; Scuseria, G. E.; Constantin, L. A.; Zhou, X.; Burke, K. Restoring the Density-Gradient Expansion for Exchange in Solids and Surfaces. Phys. Rev. Lett. 2008, $100 \quad$ (13), 136406. https://doi.org/10.1103/PhysRevLett.100.136406.

(54) Grimme, S.; Antony, J.; Ehrlich, S.; Krieg, H. A Consistent and Accurate Ab Initio Parametrization of Density Functional Dispersion Correction (DFT-D) for the 94 Elements H-Pu. J. Chem. Phys. 2010, 132 (15), 154104. https://doi.org/10.1063/1.3382344.

(55) Blöchl, P. E. Projector Augmented-Wave Method. Phys. Rev. B 1994, 50 (24), 1795317979. https://doi.org/10.1103/PhysRevB.50.17953.

(56) Hinuma, Y.; Pizzi, G.; Kumagai, Y.; Oba, F.; Tanaka, I. Band Structure Diagram Paths Based on Crystallography. Comput. Mater. Sci. 2017, 128, 140-184. https://doi.org/10.1016/J.COMMATSCI.2016.10.015.

(57) Skelton, J. M. Transformer. GitHub 2017.

(58) Atabaeva, E. Y.; Mashkov, S. A.; Popova, S. V. The Crystal Structure of a New Modification of Bi2Se3-II. Kristallografiya 1973, 18, 173-174.

(59) Zhao, J.; Liu, H.; Ehm, L.; Dong, D.; Chen, Z.; Gu, G. High-Pressure Phase Transitions, Amorphization, and Crystallization Behaviors in Bi2Se3. J. Phys. Condens. Matter 2013, 25 (12), 125602. https://doi.org/10.1088/0953-8984/25/12/125602. 
(60) Atabaeva, E. Polymorphism of $\mathrm{Bi}_{2} \mathrm{Se}_{3}$ at High Pressures and Temperatures. Sov. Phys. Solid State 1974, 15 (12), 2346-2348.

(61) Atabaeva, E. Y.; Itskevich, E. S.; Mashkov, S. A.; Popova, S. V.; Vershchagin, L. F. POLYMORPHISM OF BISMUTH TELLURIDE AT HIGH PRESSURES AND TEMPERATURES. Sov. Phys. - Solid State 1968, 10 (1), 43-46.

(62) Momma, K.; Izumi, F. VESTA 3 for Three-Dimensional Visualization of Crystal, Volumetric and Morphology Data. J. Appl. Crystallogr. 2011, 44 (6), 1272-1276. https://doi.org/10.1107/S0021889811038970.

(63) Walsh, A.; Payne, D. J.; Egdell, R. G.; Watson, G. W. Stereochemistry of Post-Transition Metal Oxides: Revision of the Classical Lone Pair Model. Chem. Soc. Rev. 2011, 40 (9), 4455-4463. https://doi.org/10.1039/C1CS15098G.

(64) Olsen, L. A.; Lpez-Solano, J.; Garca, A.; Balić-Unić, T.; Makovicky, E. Dependence of the Lone Pair of Bismuth on Coordination Environment and Pressure: An Ab Initio Study on Cu4Bi5S10 and Bi2S3. J. Solid State Chem. 2010, 183 (9), 2133-2143. https://doi.org/10.1016/J.JSSC.2010.07.022.

(65) Li, C.; Zhao, J.; Hu, Q.; Liu, Z.; Yu, Z.; Yan, H. Crystal Structure and Transporting Properties of Bi2S3 under High Pressure: Experimental and Theoretical Studies. J. Alloys Compd. 2016, 688, 329-335. https://doi.org/10.1016/J.JALLCOM.2016.06.276.

(66) Rathore, E.; Dutta, M.; Biswas, K. Influence of Periodic Table in Designing Solid-State Metal Chalcogenides for Thermoelectric Energy Conversion. J. Chem. Sci. 201913112 2019, 131 (12), 1-8. https://doi.org/10.1007/S12039-019-1704-8. 
(67) Skelton, J. M.; Burton, L. A.; Oba, F.; Walsh, A. Chemical and Lattice Stability of the Tin $\begin{array}{llllllll}\text { Sulfides. } & \text { J. Phys. Chem. } & \text { C 2017, } 121 & \text { (12), 6446-6454. }\end{array}$ https://doi.org/10.1021/acs.jpcc.6b12581.

(68) Zhao, L. D.; Zhang, B. P.; Liu, W. S.; Zhang, H. L.; Li, J. F. Enhanced Thermoelectric Properties of Bismuth Sulfide Polycrystals Prepared by Mechanical Alloying and Spark Plasma Sintering. J. $\quad$ Solid State Chem. 2008, 181 (12), 3278-3282. https://doi.org/10.1016/j.jssc.2008.08.022.

(69) Goldsmid, H. J. The Thermal Conductivity of Bismuth Telluride. Proc. Phys. Soc. Sect. B 1956, 69 (2), 203-209. https://doi.org/10.1088/0370-1301/69/2/310.

(70) Tang, J.; Skelton, J. M. Impact of Noble-Gas Filler Atoms on the Lattice Thermal Conductivity of $\mathrm{CoSb}_{3}$ Skutterudites: First-Principles Modelling. J. Phys. Condens. Matter 2021, 33 (16), 164002. https://doi.org/10.1088/1361-648x/abd8b8.

(71) Grau-Crespo, R.; Hamad, S.; Catlow, C. R. A.; de Leeuw, N. H. Symmetry-Adapted Configurational Modelling of Fractional Site Occupancy in Solids. J. Phys. Condens. Matter 2007, 19 (25), 256201. https://doi.org/10.1088/0953-8984/19/25/256201.

(72) Hasan, M. Z.; Kane, C. L. Colloquium: Topological Insulators. Rev. Mod. Phys. 2010, 82 (4), 3045. https://doi.org/10.1103/RevModPhys.82.3045.

(73) Tamura, S. I. Isotope Scattering of Dispersive Phonons in Ge. Phys. Rev. B 1983, 27 (2), 858-866. https://doi.org/10.1103/PhysRevB.27.858.

(74) Yang, H.; Savory, C. N.; Morgan, B. J.; Scanlon, D. O.; Skelton, J. M.; Walsh, A. Chemical 
Trends in the Lattice Thermal Conductivity of $\mathrm{Li}(\mathrm{Ni}, \mathrm{Mn}, \mathrm{Co}) \mathrm{O}_{2}(\mathrm{NMC})$ Battery Cathodes. Chem. Mater. 2020, 32 (17), 7542-7550. https://doi.org/10.1021/acs.chemmater.0c02908. 


\section{TOC ENTRY}

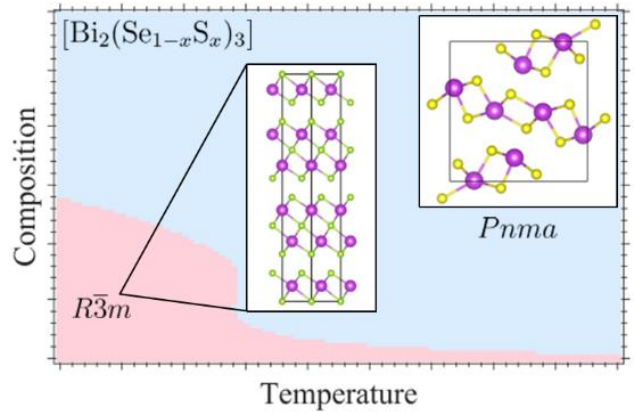

First-principles calculations are employed to study the phase stability and thermal transport of the bismuth chalcogenides $\mathrm{Bi}_{2} \mathrm{~S}_{3}, \mathrm{Bi}_{2} \mathrm{Se}_{3}, \mathrm{Bi}_{2} \mathrm{Te}_{3}$ and their alloys. 